\title{
Temporal Variations among Invasive Pneumococcal Disease Serotypes in Children and Adults in Germany (1992-2008)
}

\author{
Matthias Imöhl, ${ }^{1}$ Ralf René Reinert, ${ }^{1,2}$ and Mark van der Linden ${ }^{1}$ \\ ${ }^{1}$ National Reference Center for Streptococci, Institute of Medical Microbiology, University Hospital (RWTH), \\ 52074 Aachen, Germany \\ ${ }^{2}$ Wyeth Vaccines Research, Coeur Défense-Tour A, 110 esplanade du Général de Gaulle, 92931 Paris la Défense Cedex, France
}

Correspondence should be addressed to Matthias Imöhl, mimoehl@ukaachen.de

Received 1 April 2010; Accepted 21 April 2010

Academic Editor: Todd R. Callaway

Copyright (C) 2010 Matthias Imöhl et al. This is an open access article distributed under the Creative Commons Attribution License, which permits unrestricted use, distribution, and reproduction in any medium, provided the original work is properly cited.

Nationwide surveillance of invasive pneumococcal disease has been conducted in Germany since 1992. From 1992 to 2008, a total of 12,137 isolates from invasive pneumococcal disease were collected. Data on serotypes were available for 9,394 invasive isolates. The leading serotypes were serotypes $14(16.5 \%), 3(8.0 \%), 7 \mathrm{~F}(7.6 \%), 1(7.3 \%)$, and $23 \mathrm{~F}(6.0 \%)$. Variations in serotype distribution over the years are particularly extensive, especially concerning serotype 14 ( $\min 7.4 \%$, max $33.5 \%)$ with the highest percentages among the isolates serotyped from around 1997 to 2006. Serotypes 1 and 7F increased over the last decade. No increase was observed concerning serotype 19A. Higher pneumococcal conjugate vaccine coverages were observed among children $(7 \mathrm{v}, 57.3 \%$; $10 \mathrm{v}, 72.8 \% ; 13 \mathrm{v}, 83.5 \%)$ than among adults $(7 \mathrm{v}, 39.9 \% ; 10 \mathrm{v}, 55.5 \% ; 13 \mathrm{v}, 73.5 \%)$. The temporal variations in serotype distribution have to be kept in mind when interpreting vaccine coverages reported in epidemiological studies.

\section{Introduction}

Streptococcus pneumoniae is one of the most important pathogens in bacterial pneumonia, sepsis, and meningitis worldwide [1]. Significant temporal [2-4] and regional [57] variations among pneumococcal serotypes have been described. While the "epidemic" serogroups (serogroups 13 and 5) decreased considerably in the United States during the last century, the serogroups included in the seven-valent pneumococcal conjugate vaccine (PCV7) increased clearly [2]. A similar trend has been described in Spain, where the prevalence of PCV7 serotypes increased significantly (except serotype 4) since the start of the study in the early 1980s but then decreased considerably in the 2000s for all PCV7 serotypes except serotype 23F. Among the "epidemic" serotypes, a significant decrease of serotypes 1 and 5 has been observed in the 1980s followed by a significant increase in the late 1990s, while serotype 3 decreased continuously during the observational period. Furthermore, serotypes 6A, 7F, and 19A increased significantly since the late 1990s [4].
The widespread use of antibiotics and the increasing application of pneumococcal conjugate vaccines (a general recommendation of pneumococcal conjugate vaccination for children $<2$ years in Germany was issued at the end of July 2006) will have an impact on future changes in serotype distribution.

The NRCS has conducted surveillance for invasive pneumococcal disease in Germany since 1992 [8]. Between 1992 and 1996 surveillance was based on a limited number of laboratories in Germany on a voluntary basis [9]. In 1996, nationwide population-based surveillance of IPD in children was started [10]. This surveillance system was extended to adults in one federal state (North-Rhine Westphalia) in 2001 [11] and successively broadened to nationwide surveillance until 2009.

The aim of this study was to evaluate the serotype distribution of $S$. pneumoniae among the isolates with invasive pneumococcal disease (IPD) that were sent to the German National Reference Center for Streptococci (NRCS) between 1992 and 2008, analyze temporal trends, and calculate the 
TABLE 1

(a) Serotype distribution of IPD in Germany (1992-2008, $n=9,394)$ in all age groups.

\begin{tabular}{|c|c|c|c|c|c|c|c|c|c|c|c|c|c|c|c|c|c|c|c|}
\hline $\begin{array}{l}\text { Sero- } \\
\text { type }\end{array}$ & $\begin{array}{c}1992 \\
(\%)\end{array}$ & $\begin{array}{c}1993 \\
(\%)\end{array}$ & $\begin{array}{c}1994 \\
(\%) \\
\end{array}$ & $\begin{array}{c}1995 \\
(\%)\end{array}$ & $\begin{array}{c}1996 \\
(\%)\end{array}$ & $\begin{array}{c}1997 \\
(\%)\end{array}$ & $\begin{array}{c}1998 \\
(\%)\end{array}$ & $\begin{array}{c}1999 \\
(\%)\end{array}$ & $\begin{array}{c}2000 \\
(\%)\end{array}$ & $\begin{array}{c}2001 \\
(\%)\end{array}$ & $\begin{array}{c}2002 \\
(\%)\end{array}$ & $\begin{array}{c}2003 \\
(\%) \\
\end{array}$ & $\begin{array}{c}2004 \\
(\%)\end{array}$ & $\begin{array}{c}2005 \\
(\%)\end{array}$ & $\begin{array}{c}2006 \\
(\%)\end{array}$ & $\begin{array}{c}2007 \\
(\%) \\
\end{array}$ & $\begin{array}{c}2008 \\
(\%)\end{array}$ & $\begin{array}{c}\text { Total } \\
(\%)\end{array}$ & $\begin{array}{c}\text { Total } \\
(n)\end{array}$ \\
\hline 14 & 8.8 & 12.6 & 7.4 & 8.5 & 13.5 & 16.5 & 17.5 & 29.8 & 33.5 & 25.2 & 21.3 & 22.8 & 23.0 & 18.8 & 19.3 & 13.3 & 8.9 & 16.5 & 1,549 \\
\hline - & & 2.9 & 3.7 & 6.0 & 9.4 & 4.2 & 2.7 & 4.3 & 2.8 & 4.0 & 6.6 & 6.7 & 4.9 & 7.5 & 6.4 & 10.5 & 11.7 & 8.0 & 755 \\
\hline $7 \mathrm{~F}$ & & 2.9 & & & 5.2 & & & & & 3.7 & & & & & & & 10.4 & 7.6 & 718 \\
\hline 1 & & 5.8 & 1.2 & 4.3 & 3.1 & 9.4 & 5.8 & 5.5 & 6.7 & 6.4 & 4.3 & 5.3 & 6.9 & 6.9 & 7.9 & 8.5 & 8.8 & 7.3 & 690 \\
\hline 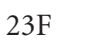 & & 9.7 & 6.2 & 8.5 & & 6.1 & 8.5 & & & 7.7 & & & & 6.9 & & & 4.3 & 6.0 & 559 \\
\hline 4 & & 11.7 & 4.9 & & & & & & & & & & & & & & 4.6 & 5.4 & 507 \\
\hline 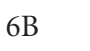 & & 7.8 & . & 6.8 & 6.3 & 8.0 & 5.8 & 6.8 & & 7.7 & 6.8 & & & & & & & & 449 \\
\hline $9 \mathrm{~F}$ & 6.7 & 1.9 & 9.9 & 6.8 & 3.1 & & 4.9 & & & 6.9 & & 3.8 & & 5.4 & & & 3.4 & & 447 \\
\hline $9 \mathrm{~V}$ & & 1.0 & 8.6 & 6.0 & & 2.8 & 3.1 & & & 2.2 & & & & 4.8 & & & 3.6 & 4.5 & 425 \\
\hline A & & 5.8 & & & & & 5.4 & & & 3.5 & & & & & & & & & 401 \\
\hline $18 \mathrm{C}$ & & 4.9 & & 1.7 & & & 6.7 & & & 4.5 & & 2.9 & & 3.4 & & & 3.0 & & 326 \\
\hline $19 \mathrm{~A}$ & 0.8 & 2.9 & 1.2 & 1.7 & 5.2 & 2.4 & 5.8 & & & 3.0 & & & & 2.9 & & & 4.2 & & 320 \\
\hline $22 \mathrm{~F}$ & & 2.9 & & & & & 0.9 & & & & & & & & & & & & 208 \\
\hline 8 & & 1.9 & & & 2.1 & & & 0.0 & & 1.5 & & 2.1 & & 2.5 & 2.2 & & & & 208 \\
\hline $10 \mathrm{~A}$ & 1.7 & 1.0 & 3.7 & 2.6 & 2.1 & & 1.3 & & & 1.7 & & 0.6 & & 1.5 & & & & & 172 \\
\hline $9 \mathrm{~N}$ & & & & & & & 0.9 & & & & & & & & & & & & 172 \\
\hline $11 \mathrm{~A}$ & & & & & & & & & & & & & & & & & & & 149 \\
\hline $12 \mathrm{~F}$ & & 1.9 & 2.5 & 1.7 & 6.3 & & 1.3 & & & 2.2 & & & & 0.4 & & & 1.7 & & 118 \\
\hline $4 \mathrm{~F}$ & & 1.0 & & & & & & & & 2.0 & & & & & & & & & 116 \\
\hline & & & & & & & & & & & & & & & & & & & 38 \\
\hline $15 \mathrm{~B}$ & 0.8 & 1.9 & 0.0 & 0.0 & 1.0 & 0.0 & 0.9 & 0.9 & & 0.0 & & 0.3 & 0.9 & 1.1 & 0.9 & 1.0 & & & 83 \\
\hline $35 \mathrm{~F}$ & 0.8 & 0.0 & 1.2 & 0.9 & 0.0 & 0.5 & 0.4 & 0.9 & 0.8 & 0.5 & & & 0.0 & 0.4 & & 0.7 & 1.8 & & 74 \\
\hline & & & & & & & & & & & & & & & & & & & 68 \\
\hline 38 & & & & & & & & & & & & & & & & & & & 61 \\
\hline 5 & & 0.0 & & 0.9 & 2.1 & 1.4 & & & & 0.2 & & 0.2 & 0.4 & 0.4 & & & & 0.6 & 55 \\
\hline & & & & & & & & & & & & & & & & & & & 53 \\
\hline $15 \mathrm{~A}$ & & & & & & & & & & & & & & & & & & & 48 \\
\hline $9 \mathrm{~A}$ & 0.4 & 1.0 & 1.2 & 0.9 & 2.1 & 0.9 & 0.9 & 0 & 0.8 & 3.5 & 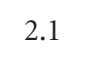 & 0.6 & 0.0 & 0.4 & & 0.2 & 0.0 & 0.5 & 47 \\
\hline 20 & & & & & & & & & & & & & & & & & & & 43 \\
\hline NT & & & & & & & & & & & & & & & & & & & 10 \\
\hline $17 \mathrm{~F}$ & & & & 0.9 & & & & & 0.0 & 0.0 & & 0.3 & & 0.8 & & 0.4 & & & 39 \\
\hline $16 \mathrm{~F}$ & & & & 1.7 & 1.0 & & 0.0 & & & 1.0 & & & 0.4 & 0.2 & & & 0.4 & & 34 \\
\hline $33 \mathrm{~A}$ & & & & & & & & & & & & & & & & & & & 30 \\
\hline 31 & & & & & & & & & & & & & & & & & & & 26 \\
\hline $18 \mathrm{~A}$ & 0.0 & 0.0 & & 0.0 & & 0.0 & 0.0 & & & 0.5 & & 0.6 & & 0.2 & & 0.3 & 0.2 & 0.2 & 22 \\
\hline 34 & & & & & & & & & & & & & & & & & & & 22 \\
\hline roug & & & & & & & & & & & & & & & & & & & 20 \\
\hline $23 \mathrm{~B}$ & 0.0 & 1.0 & & 0.0 & 0.0 & & 0.4 & & 0.0 & 0.5 & & 0.2 & & 0.1 & & 0.1 & 0.2 & 0.2 & 17 \\
\hline 9L & & & & & & & & & & & & & & & & & 0.0 & & 17 \\
\hline 13 & & & & & & & & & & & & & & & & & & & 15 \\
\hline $18 \mathrm{~F}$ & 0.4 & 1.9 & 0.0 & 0.9 & 0.0 & 0.5 & 0.0 & 0.9 & 0.0 & 0.0 & 0.2 & 0.2 & 0.0 & 0.1 & 0.0 & 0.3 & 0.0 & 0.2 & 15 \\
\hline 2 & & & & & & & & & & & & & & & & & 0.0 & 0.1 & 13 \\
\hline $12 \mathrm{~A}$ & & & & 0.0 & & & 0.0 & & & 0.0 & & 0.0 & & & & 0.2 & 0.0 & 0.1 & 12 \\
\hline $28 \mathrm{~A}$ & & 0.0 & & 0.0 & 0.0 & & 0.0 & .0 & 0.4 & 0.0 & 0.4 & 0.2 & 0.0 & 0.1 & 0.1 & 0.2 & 0.0 & 0.1 & 12 \\
\hline
\end{tabular}


(a) Continued.

\begin{tabular}{|c|c|c|c|c|c|c|c|c|c|c|c|c|c|c|c|c|c|c|c|}
\hline $\begin{array}{l}\text { Sero- } \\
\text { type }\end{array}$ & $\begin{array}{c}1992 \\
(\%)\end{array}$ & $\begin{array}{c}1993 \\
(\%)\end{array}$ & $\begin{array}{c}1994 \\
(\%)\end{array}$ & $\begin{array}{c}1995 \\
(\%)\end{array}$ & $\begin{array}{c}1996 \\
(\%)\end{array}$ & $\begin{array}{c}1997 \\
(\%)\end{array}$ & $\begin{array}{c}1998 \\
(\%)\end{array}$ & $\begin{array}{c}1999 \\
(\%)\end{array}$ & $\begin{array}{c}2000 \\
(\%)\end{array}$ & $\begin{array}{c}2001 \\
(\%)\end{array}$ & $\begin{array}{c}2002 \\
(\%)\end{array}$ & $\begin{array}{c}2003 \\
(\%) \\
\end{array}$ & $\begin{array}{c}2004 \\
(\%)\end{array}$ & $\begin{array}{c}2005 \\
(\%)\end{array}$ & $\begin{array}{c}2006 \\
(\%)\end{array}$ & $\begin{array}{c}2007 \\
(\%)\end{array}$ & $\begin{array}{c}2008 \\
(\%)\end{array}$ & $\begin{array}{l}\text { Total } \\
(\%)\end{array}$ & $\begin{array}{c}\text { Total } \\
(n)\end{array}$ \\
\hline $35 B$ & 0.0 & 0.0 & 0.0 & 0.0 & 0.0 & 0.0 & 0.0 & 0.0 & 0.0 & 0.0 & 0.2 & 0.0 & 0.2 & 0.0 & 0.0 & 0 & 0.2 & 0.1 & 11 \\
\hline $10 \mathrm{~B}$ & 0.0 & 0.0 & 0.0 & 0.0 & 0.0 & 0.0 & 0.4 & 0.0 & 0.0 & 0.0 & 0.0 & 0.0 & 0.2 & 0.2 & 0.0 & 0.2 & 0.1 & 0.1 & 10 \\
\hline Others ${ }^{\star}$ & 2.9 & 1.9 & 2.5 & 7.7 & 3.1 & 0.5 & 4.9 & 1.7 & 1.2 & 1.5 & 0.6 & 1.6 & 0.6 & 1.3 & 0.3 & 1.7 & 0.9 & 1.4 & 130 \\
\hline otal & 100 & 100 & 00 & 100 & 10 & 100 & 100 & 100 & 100 & 100 & 100 & 100 & 10 & 100 & 100 & 10 & 10 & 100 & 9,394 \\
\hline $\begin{array}{l}\text { Jot } \\
\text { ero- } \\
\text { yped }\end{array}$ & 2.5 & 7.8 & 6.0 & 7.0 & 7.7 & 40.8 & 40.5 & 43.9 & 38.2 & 39.4 & 29.9 & 1.5 & 19.1 & 1.5 & 0.0 & 0.1 & 0.0 & 2.6 & 2,743 \\
\hline
\end{tabular}

NT: nontypeable; $n$ : number of isolates tested.

Others $^{\star}$ includes the serotypes (number of isolates): 15F (9), 18B (9), 12B (8), 7C (8), 33B (7), 10F (6), 11B (6), 11F (6), 35A (6), 19C (5), 29 (5), 37 (5), 21 (4), 24A (4), 28F (4), 35C (4), 19B (3), 22A (3), 36 (3), 45 (3), 7A (3), 24B (2), 25F (2), 39 (2), 48 (2), 6 (2), 7B (2), 12 (1), $17 \mathrm{~A}(1), 18$ (1), 19 (1), 23 (1), 35 (1), 9 (1)

(b) Serotype distribution of IPD in Germany (1992-2008, $n=2,948)$ in children ( $<16$ years).

\begin{tabular}{|c|c|c|c|c|c|c|c|c|c|c|c|c|c|c|c|c|c|c|c|}
\hline $\begin{array}{l}\text { Sero- } \\
\text { type }\end{array}$ & $\begin{array}{c}1992 \\
(\%)\end{array}$ & $\begin{array}{c}1993 \\
(\%)\end{array}$ & $\begin{array}{c}1994 \\
(\%)\end{array}$ & $\begin{array}{c}1995 \\
(\%)\end{array}$ & $\begin{array}{c}1996 \\
(\%)\end{array}$ & $\begin{array}{c}1997 \\
(\%)\end{array}$ & $\begin{array}{c}1998 \\
(\%)\end{array}$ & $\begin{array}{c}1999 \\
(\%)\end{array}$ & $\begin{array}{c}2000 \\
(\%)\end{array}$ & $\begin{array}{c}2001 \\
(\%)\end{array}$ & $\begin{array}{c}2002 \\
(\%)\end{array}$ & $\begin{array}{c}2003 \\
(\%)\end{array}$ & $\begin{array}{c}2004 \\
(\%)\end{array}$ & $\begin{array}{c}2005 \\
(\%)\end{array}$ & $\begin{array}{c}2006 \\
(\%)\end{array}$ & $\begin{array}{c}2007 \\
(\%)\end{array}$ & $\begin{array}{c}2008 \\
(\%)\end{array}$ & $\begin{array}{l}\text { Total } \\
(\%)\end{array}$ & $\begin{array}{c}\text { Total } \\
(n)\end{array}$ \\
\hline 14 & 12.8 & 6.9 & 14.3 & 5.9 & 16.7 & 15.2 & 18.0 & 26.9 & 31.5 & 25.5 & 26.7 & 27.0 & 30.6 & 26.3 & 25.4 & 13.9 & 5.6 & 22.5 & 664 \\
\hline 1 & 2.1 & 3.4 & 3.6 & 0.0 & 0.0 & 9.5 & 5.6 & 6.6 & 8.0 & 8.4 & 5.4 & 4.5 & 6.0 & 7.6 & 10.5 & 12.5 & 12.9 & 7.9 & 234 \\
\hline $6 \mathrm{~B}$ & 6.4 & 13.8 & 0.0 & 11.8 & 8.3 & 8.9 & 6.2 & 7.7 & 3.5 & 7.5 & 8.9 & 7.8 & 7.2 & 10.8 & 6.6 & 7.0 & 4.3 & 7.3 & 216 \\
\hline $19 \mathrm{~F}$ & 8.5 & 3.4 & 7.1 & 23.5 & 0.0 & 6.3 & 6.8 & 8.8 & 8.0 & 9.6 & 5.4 & 5.7 & 6.8 & 7.3 & 8.0 & 8.4 & 4.7 & 7.2 & 213 \\
\hline $23 \mathrm{~F}$ & 10.6 & 3.4 & 7.1 & 11.8 & 16.7 & 7.0 & 7.5 & 8.2 & 5.5 & 7.9 & 10.5 & 6.1 & 4.9 & 6.6 & 8.7 & 6.2 & 2.6 & 6.9 & 204 \\
\hline $7 F$ & 2.1 & 3.4 & 0.0 & 5.9 & 0.0 & 7.6 & 3.7 & 2.2 & 5.5 & 4.6 & 5.8 & 8.6 & 7.9 & 6.0 & 7.7 & 9.9 & 13.8 & 6.9 & 204 \\
\hline $18 \mathrm{C}$ & 8.5 & 10.3 & 17.9 & 0.0 & 0.0 & 8.9 & 8.7 & 4.9 & 8.0 & 6.3 & 5.8 & 5.7 & 3.8 & 5.4 & 6.6 & 5.1 & 5.6 & 6.2 & 182 \\
\hline $6 \mathrm{~A}$ & 14.9 & 20.7 & 3.6 & 5.9 & 0.0 & 5.1 & 5.6 & 2.7 & 4.5 & 3.8 & 4.7 & 4.9 & 3.8 & 3.5 & 1.4 & 4.8 & 5.6 & 4.4 & 130 \\
\hline $9 \mathrm{~V}$ & 4.3 & 3.4 & 14.3 & 11.8 & 8.3 & 2.5 & 2.5 & 7.1 & 4.0 & 2.1 & 2.3 & 4.1 & 4.2 & 5.1 & 3.1 & 3.3 & 1.3 & 3.7 & 108 \\
\hline 4 & 6.4 & 10.3 & 7.1 & 11.8 & 8.3 & 5.1 & 7.5 & 3.8 & 4.0 & 2.1 & 3.5 & 3.7 & 3.0 & 2.8 & 3.1 & 2.2 & 0.4 & 3.5 & 102 \\
\hline 3 & 4.3 & 3.4 & 3.6 & 5.9 & 0.0 & 3.8 & 1.9 & 3.8 & 3.0 & 2.9 & 3.1 & 3.3 & 1.5 & 1.9 & 2.4 & 5.9 & 5.6 & 3.3 & 96 \\
\hline $19 \mathrm{~A}$ & 0.0 & 3.4 & 0.0 & 0.0 & 8.3 & 1.9 & 6.2 & 2.2 & 3.5 & 2.5 & 1.6 & 2.0 & 3.0 & 2.5 & 3.8 & 4.0 & 4.7 & 3.1 & 90 \\
\hline $10 \mathrm{~A}$ & 2.1 & 0.0 & 0.0 & 0.0 & 0.0 & 0.6 & 0.0 & 1.1 & 1.5 & 2.1 & 1.6 & 0.4 & 2.3 & 1.9 & 2.8 & 2.2 & 3.4 & 1.7 & 51 \\
\hline $24 \mathrm{~F}$ & 4.3 & 3.4 & 0.0 & 0.0 & 0.0 & 0.0 & 2.5 & 1.6 & 1.0 & 2.1 & 0.8 & 2.5 & 1.9 & 0.6 & 1.4 & 1.1 & 2.2 & 1.5 & 44 \\
\hline $15 \mathrm{~B}$ & 0.0 & 0.0 & 0.0 & 0.0 & 0.0 & 0.0 & 0.6 & 1.1 & 1.0 & 0.0 & 0.4 & 0.4 & 0.8 & 1.3 & 0.7 & 2.2 & 2.2 & 0.9 & 26 \\
\hline $15 \mathrm{C}$ & 2.1 & 0.0 & 0.0 & 0.0 & 0.0 & 4.4 & 0.0 & 0.0 & 0.0 & 0.0 & 0.4 & 0.8 & 0.4 & 1.3 & 1.4 & 0.4 & 2.2 & 0.9 & 26 \\
\hline $9 \mathrm{~N}$ & 0.0 & 0.0 & 0.0 & 0.0 & 0.0 & 0.0 & 0.6 & 1.1 & 1.5 & 0.8 & 0.4 & 1.6 & 1.5 & 0.3 & 1.0 & 0.4 & 1.7 & 0.9 & 26 \\
\hline 38 & 2.1 & 0.0 & 3.6 & 0.0 & 0.0 & 0.6 & 0.6 & 0.0 & 0.5 & 0.4 & 0.8 & 0.4 & 0.8 & 0.3 & 0.0 & 0.7 & 3.9 & 0.8 & 23 \\
\hline $15 \mathrm{~A}$ & 0.0 & 0.0 & 0.0 & 0.0 & 0.0 & 0.0 & 1.2 & 1.6 & 0.0 & 0.4 & 1.2 & 1.6 & 0.8 & 0.6 & 1.0 & 0.0 & 0.4 & 0.7 & 21 \\
\hline $22 \mathrm{~F}$ & 0.0 & 0.0 & 7.1 & 0.0 & 0.0 & 0.6 & 0.6 & 0.0 & 0.0 & 0.8 & 1.2 & 1.2 & 0.8 & 0.6 & 0.3 & 0.4 & 1.3 & 0.7 & 21 \\
\hline $9 \mathrm{~A}$ & 0.0 & 0.0 & 0.0 & 0.0 & 0.0 & 1.3 & 0.6 & 0.0 & 1.0 & 3.8 & 1.9 & 0.8 & 0.0 & 0.0 & 0.0 & 0.0 & 0.0 & 0.7 & 21 \\
\hline 5 & 0.0 & 0.0 & 3.6 & 0.0 & 8.3 & 1.9 & 1.2 & 1.1 & 0.0 & 0.0 & 0.8 & 0.0 & 0.8 & 0.9 & 0.3 & 0.0 & 0.9 & 0.6 & 19 \\
\hline 8 & 0.0 & 0.0 & 0.0 & 0.0 & 0.0 & 0.6 & 1.2 & 0.0 & 0.5 & 0.4 & 1.2 & 0.4 & 0.8 & 0.9 & 0.7 & 0.4 & 0.4 & 0.6 & 18 \\
\hline $33 \mathrm{~F}$ & 0.0 & 0.0 & 0.0 & 0.0 & 0.0 & 1.3 & 1.9 & 0.5 & 0.0 & 0.4 & 1.2 & 0.8 & 0.4 & 0.0 & 0.0 & 0.4 & 1.3 & 0.6 & 17 \\
\hline $12 \mathrm{~F}$ & 2.1 & 0.0 & 3.6 & 0.0 & 0.0 & 0.0 & 0.6 & 1.1 & 0.0 & 1.7 & 0.0 & 0.0 & 0.8 & 0.0 & 0.0 & 0.4 & 1.7 & 0.5 & 16 \\
\hline $11 \mathrm{~A}$ & 0.0 & 0.0 & 0.0 & 0.0 & 8.3 & 1.9 & 0.0 & 0.5 & 0.0 & 0.0 & 0.4 & 1.2 & 0.0 & 0.0 & 0.3 & 1.1 & 0.9 & 0.5 & 15 \\
\hline $23 \mathrm{~A}$ & 2.1 & 0.0 & 0.0 & 0.0 & 0.0 & 0.6 & 1.2 & 0.0 & 0.5 & 0.0 & 0.4 & 0.4 & 0.0 & 0.0 & 0.7 & 0.4 & 1.7 & 0.5 & 14 \\
\hline $18 \mathrm{~A}$ & 0.0 & 0.0 & 0.0 & 0.0 & 0.0 & 0.0 & 0.0 & 0.0 & 0.0 & 0.8 & 0.8 & 0.8 & 0.4 & 0.0 & 0.0 & 0.4 & 0.9 & 0.3 & 10 \\
\hline $35 \mathrm{~F}$ & 0.0 & 0.0 & 0.0 & 0.0 & 0.0 & 0.0 & 0.6 & 0.5 & 1.0 & 0.0 & 0.0 & 0.0 & 0.0 & 0.3 & 0.3 & 0.4 & 1.3 & 0.3 & 10 \\
\hline NT & 0.0 & 0.0 & 0.0 & 0.0 & 0.0 & 0.0 & 0.0 & 0.5 & 0.0 & 0.0 & 0.0 & 0.0 & 1.5 & 0.3 & 0.0 & 0.7 & 0.9 & 0.3 & 10 \\
\hline $33 \mathrm{~A}$ & 0.0 & 0.0 & 0.0 & 0.0 & 0.0 & 0.0 & 0.0 & 0.0 & 0.0 & 0.0 & 0.0 & 0.0 & 0.4 & 1.3 & 0.7 & 0.4 & 0.4 & 0.3 & 9 \\
\hline rough & 0.0 & 3.4 & 0.0 & 0.0 & 0.0 & 0.0 & 0.0 & 0.0 & 0.5 & 0.8 & 0.8 & 1.2 & 0.0 & 0.0 & 0.0 & 0.0 & 0.0 & 0.3 & 9 \\
\hline $17 \mathrm{~F}$ & 0.0 & 0.0 & 0.0 & 0.0 & 0.0 & 0.0 & 1.2 & 0.0 & 0.0 & 0.0 & 0.0 & 0.0 & 0.8 & 0.3 & 0.0 & 0.4 & 0.9 & 0.3 & 8 \\
\hline $16 \mathrm{~F}$ & 0.0 & 0.0 & 0.0 & 0.0 & 0.0 & 0.6 & 0.0 & 0.0 & 0.0 & 0.8 & 0.4 & 0.0 & 0.0 & 0.3 & 0.0 & 0.0 & 0.9 & 0.2 & 7 \\
\hline $18 \mathrm{~B}$ & 0.0 & 0.0 & 0.0 & 0.0 & 8.3 & 0.0 & 1.2 & 0.5 & 0.5 & 0.0 & 0.0 & 0.0 & 0.0 & 0.6 & 0.0 & 0.0 & 0.0 & 0.2 & 7 \\
\hline
\end{tabular}


(b) Continued.

\begin{tabular}{lccccccccccccccccccc}
\hline $\begin{array}{l}\text { Sero- } \\
\text { type }\end{array}$ & $\begin{array}{c}1992 \\
(\%)\end{array}$ & $\begin{array}{c}1993 \\
(\%)\end{array}$ & $\begin{array}{c}1994 \\
(\%)\end{array}$ & $\begin{array}{c}1995 \\
(\%)\end{array}$ & $\begin{array}{c}1996 \\
(\%)\end{array}$ & $\begin{array}{c}1997 \\
(\%)\end{array}$ & $\begin{array}{c}1998 \\
(\%)\end{array}$ & $\begin{array}{c}1999 \\
(\%)\end{array}$ & $\begin{array}{c}2000 \\
(\%)\end{array}$ & $\begin{array}{c}2001 \\
(\%)\end{array}$ & $\begin{array}{c}2002 \\
(\%)\end{array}$ & $\begin{array}{c}2003 \\
(\%)\end{array}$ & $\begin{array}{c}2004 \\
(\%)\end{array}$ & $\begin{array}{c}2005 \\
(\%)\end{array}$ & $\begin{array}{c}2006 \\
(\%)\end{array}$ & $\begin{array}{c}2007 \\
(\%)\end{array}$ & $\begin{array}{c}2008 \\
(\%)\end{array}$ & $\begin{array}{c}\text { Total } \\
(\%)\end{array}$ & $\begin{array}{c}\text { Total } \\
(n)\end{array}$ \\
\hline $23 \mathrm{~B}$ & 0.0 & 3.4 & 0.0 & 0.0 & 0.0 & 0.6 & 0.6 & 0.0 & 0.0 & 0.0 & 0.0 & 0.4 & 0.4 & 0.0 & 0.0 & 0.0 & 0.4 & 0.2 & 6 \\
34 & 2.1 & 0.0 & 0.0 & 0.0 & 0.0 & 0.0 & 0.6 & 0.0 & 0.0 & 0.0 & 0.0 & 0.0 & 0.4 & 0.3 & 0.0 & 0.7 & 0.0 & 0.2 & 6 \\
$18 \mathrm{~F}$ & 0.0 & 0.0 & 0.0 & 0.0 & 0.0 & 0.6 & 0.0 & 1.1 & 0.0 & 0.0 & 0.4 & 0.0 & 0.0 & 0.0 & 0.0 & 0.4 & 0.0 & 0.2 & 5 \\
20 & 0.0 & 0.0 & 0.0 & 0.0 & 0.0 & 0.0 & 0.0 & 0.5 & 0.0 & 0.0 & 0.0 & 0.4 & 0.4 & 0.3 & 0.0 & 0.4 & 0.0 & 0.2 & 5 \\
9L & 0.0 & 0.0 & 0.0 & 0.0 & 0.0 & 0.0 & 0.0 & 0.0 & 0.0 & 0.4 & 0.8 & 0.4 & 0.0 & 0.0 & 0.3 & 0.0 & 0.0 & 0.2 & 5 \\
Others & 2.1 & 3.4 & 3.6 & 5.9 & 8.3 & 2.5 & 2.5 & 1.6 & 1.5 & 0.8 & 0.8 & 0.4 & 1.5 & 1.6 & 0.3 & 3.3 & 3.0 & 1.7 & 50 \\
Total & 100 & 100 & 100 & 100 & 100 & 100 & 100 & 100 & 100 & 100 & 100 & 100 & 100 & 100 & 100 & 100 & 100 & 100 & 2,948 \\
\hline $\begin{array}{l}\text { Not } \\
\text { sero- }\end{array}$ & 7.8 & 17.1 & 3.4 & 34.6 & 61.3 & 7.1 & 2.4 & 3.2 & 3.8 & 0.0 & 0.4 & 0.8 & 0.0 & 0.0 & 0.0 & 0.0 & 0.0 & 2.4 & 72 \\
typed & & & & & & & & & & & & & & & & & & \\
\hline
\end{tabular}

NT: nontypeable; $n$ : number of isolates tested.

Others ${ }^{\star}$ includes the serotypes (number of isolates): 28A (4), 11B (3), 12A (3), 12B (3), 13 (3), 33B (3), 35B (3), 15F (2), 19C (2), 21 (2), 24A (2), 28F (2), 29 (2), 35A (2), 37 (2), 7A (2), 10F (1), 19B (1), 2 (1), 31 (1), 35 (1), 35C (1), 36 (1), 39 (1), 6 (1), 7C (1).

(c) Serotype distribution of IPD in Germany (1992-2008, $n=6,446)$ in adults ( $\geq 16$ years).

\begin{tabular}{|c|c|c|c|c|c|c|c|c|c|c|c|c|c|c|c|c|c|c|c|}
\hline $\begin{array}{l}\text { Sero- } \\
\text { type }\end{array}$ & $\begin{array}{c}1992 \\
(\%)\end{array}$ & $\begin{array}{c}1993 \\
(\%)\end{array}$ & $\begin{array}{c}1994 \\
(\%)\end{array}$ & $\begin{array}{c}1995 \\
(\%)\end{array}$ & $\begin{array}{c}1996 \\
(\%)\end{array}$ & $\begin{array}{c}1997 \\
(\%)\end{array}$ & $\begin{array}{c}1998 \\
(\%)\end{array}$ & $\begin{array}{c}1999 \\
(\%)\end{array}$ & $\begin{array}{c}2000 \\
(\%)\end{array}$ & $\begin{array}{c}2001 \\
(\%)\end{array}$ & $\begin{array}{c}2002 \\
(\%)\end{array}$ & $\begin{array}{c}2003 \\
(\%) \\
\end{array}$ & $\begin{array}{c}2004 \\
(\%)\end{array}$ & $\begin{array}{c}2005 \\
(\%)\end{array}$ & $\begin{array}{c}2006 \\
(\%) \\
\end{array}$ & $\begin{array}{c}2007 \\
(\%) \\
\end{array}$ & $\begin{array}{c}2008 \\
(\%) \\
\end{array}$ & $\begin{array}{c}\text { Total } \\
(\%)\end{array}$ & $\begin{array}{c}\text { Total } \\
(n)\end{array}$ \\
\hline 14 & 7.8 & 14.9 & 3.8 & 9.0 & 13.1 & 20.4 & 16.1 & 39.6 & 40.7 & 24.8 & 15.1 & 20.1 & 15.6 & 14.8 & 16.6 & 13.2 & 9.4 & 13.7 & 885 \\
\hline 3 & 7.8 & 2.7 & 3.8 & 6.0 & 10.7 & 5.6 & 4.8 & 5.7 & 1.9 & 5.5 & 10.7 & 8.9 & 8.1 & 10.5 & 8.3 & 11.2 & 12.5 & 10.2 & 659 \\
\hline $7 F$ & 7.8 & 2.7 & 7.5 & 4.0 & 6.0 & 7.4 & 0.0 & 1.9 & 0.0 & 2.4 & 5.8 & 6.5 & 10.0 & 6.6 & 8.1 & 8.5 & 9.9 & 8.0 & 514 \\
\hline 1 & 8.8 & 6.8 & 0.0 & 5.0 & 3.6 & 9.3 & 6.5 & 1.9 & 1.9 & 3.6 & 3.1 & 5.7 & 7.8 & 6.5 & 6.7 & 7.8 & 8.3 & 7.1 & 456 \\
\hline 4 & 7.8 & 12.2 & 3.8 & 4.0 & 1.2 & 5.6 & 6.5 & 1.9 & 3.7 & 5.5 & 5.3 & 9.9 & 6.7 & 7.7 & 7.6 & 6.1 & 5.2 & 6.3 & 405 \\
\hline $23 \mathrm{~F}$ & 7.8 & 12.2 & 5.7 & 8.0 & 8.3 & 3.7 & 11.3 & 11.3 & 9.3 & 7.3 & 6.2 & 4.4 & 4.8 & 7.0 & 5.9 & 4.7 & 4.5 & 5.5 & 355 \\
\hline $9 \mathrm{~V}$ & 4.1 & 0.0 & 5.7 & 5.0 & 8.3 & 3.7 & 4.8 & 3.8 & 11.1 & 2.4 & 4.4 & 6.0 & 7.0 & 4.6 & 6.1 & 5.4 & 3.9 & 4.9 & 317 \\
\hline $6 \mathrm{~A}$ & 4.7 & 0.0 & 5.7 & 9.0 & 3.6 & 5.6 & 4.8 & 1.9 & 3.7 & 3.0 & 2.2 & 4.4 & 4.1 & 2.4 & 3.8 & 4.9 & 4.5 & 4.2 & 271 \\
\hline $19 \mathrm{~F}$ & 6.2 & 1.4 & 11.3 & 4.0 & 3.6 & 1.9 & 0.0 & 3.8 & 3.7 & 3.0 & 4.9 & 2.6 & 4.1 & 4.4 & 2.9 & 3.8 & 3.3 & 3.6 & 234 \\
\hline $6 \mathrm{~B}$ & 2.6 & 5.4 & 11.3 & 6.0 & 6.0 & 5.6 & 4.8 & 3.8 & 1.9 & 7.9 & 4.4 & 4.4 & 3.3 & 5.1 & 4.0 & 3.2 & 2.3 & 3.6 & 233 \\
\hline $19 \mathrm{~A}$ & 1.0 & 2.7 & 1.9 & 2.0 & 4.8 & 3.7 & 4.8 & 7.5 & 7.4 & 3.6 & 1.3 & 1.3 & 2.2 & 3.1 & 4.5 & 4.0 & 4.1 & 3.6 & 230 \\
\hline 8 & 2.6 & 2.7 & 0.0 & 1.0 & 2.4 & 0.0 & 0.0 & 0.0 & 0.0 & 3.0 & 3.6 & 3.1 & 3.3 & 3.4 & 2.9 & 2.8 & 3.4 & 2.9 & 190 \\
\hline $22 \mathrm{~F}$ & 1.6 & 4.1 & 1.9 & 0.0 & 2.4 & 0.0 & 1.6 & 0.0 & 0.0 & 0.6 & 0.9 & 0.8 & 2.2 & 4.3 & 4.1 & 3.3 & 3.3 & 2.9 & 187 \\
\hline $9 \mathrm{~N}$ & 2.6 & 1.4 & 1.9 & 4.0 & 1.2 & 5.6 & 1.6 & 1.9 & 1.9 & 1.8 & 3.1 & 1.0 & 1.1 & 2.0 & 1.9 & 2.3 & 2.7 & 2.3 & 146 \\
\hline $18 \mathrm{C}$ & 3.6 & 2.7 & 0.0 & 2.0 & 1.2 & 0.0 & 1.6 & 0.0 & 5.6 & 1.8 & 2.7 & 1.0 & 3.3 & 2.4 & 2.2 & 1.8 & 2.7 & 2.2 & 144 \\
\hline $11 \mathrm{~A}$ & 3.1 & 2.7 & 1.9 & 2.0 & 3.6 & 0.0 & 1.6 & 1.9 & 0.0 & 0.6 & 0.9 & 2.1 & 3.0 & 2.0 & 1.4 & 1.8 & 2.7 & 2.1 & 134 \\
\hline $10 \mathrm{~A}$ & 1.6 & 1.4 & 5.7 & 3.0 & 2.4 & 3.7 & 4.8 & 0.0 & 1.9 & 1.2 & 3.6 & 0.8 & 2.2 & 1.4 & 1.4 & 1.8 & 2.0 & 1.9 & 121 \\
\hline $12 \mathrm{~F}$ & 2.6 & 2.7 & 1.9 & 2.0 & 7.1 & 5.6 & 3.2 & 1.9 & 0.0 & 3.0 & 2.2 & 2.9 & 0.7 & 0.7 & 0.6 & 1.1 & 1.7 & 1.6 & 102 \\
\hline $23 \mathrm{~A}$ & 0.5 & 1.4 & 1.9 & 0.0 & 0.0 & 1.9 & 1.6 & 0.0 & 1.9 & 1.2 & 1.3 & 1.0 & 0.4 & 0.7 & 0.8 & 1.1 & 1.7 & 1.1 & 74 \\
\hline $24 \mathrm{~F}$ & 1.6 & 0.0 & 1.9 & 1.0 & 1.2 & 1.9 & 0.0 & 0.0 & 0.0 & 1.8 & 0.4 & 0.5 & 1.1 & 0.7 & 1.1 & 0.8 & 1.7 & 1.1 & 72 \\
\hline $35 \mathrm{~F}$ & 1.0 & 0.0 & 1.9 & 1.0 & 0.0 & 1.9 & 0.0 & 1.9 & 0.0 & 1.2 & 0.0 & 0.5 & 0.0 & 0.5 & 0.6 & 0.8 & 1.9 & 1.0 & 64 \\
\hline $15 B$ & 1.0 & 2.7 & 0.0 & 0.0 & 1.2 & 0.0 & 1.6 & 0.0 & 1.9 & 0.0 & 0.9 & 0.3 & 1.1 & 1.0 & 1.0 & 0.8 & 1.1 & 0.9 & 57 \\
\hline $33 \mathrm{~F}$ & 1.6 & 1.4 & 1.9 & 1.0 & 0.0 & 0.0 & 1.6 & 1.9 & 0.0 & 0.6 & 0.0 & 1.0 & 1.5 & 1.0 & 0.3 & 0.6 & 0.9 & 0.8 & 51 \\
\hline 20 & 1.0 & 2.7 & 3.8 & 0.0 & 0.0 & 0.0 & 0.0 & 0.0 & 0.0 & 0.0 & 0.0 & 0.8 & 0.7 & 1.0 & 0.5 & 0.6 & 0.4 & 0.6 & 38 \\
\hline 38 & 0.0 & 0.0 & 1.9 & 0.0 & 0.0 & 1.9 & 0.0 & 0.0 & 0.0 & 0.6 & 0.4 & 0.8 & 0.7 & 0.2 & 0.5 & 0.3 & 1.1 & 0.6 & 38 \\
\hline 5 & 1.6 & 0.0 & 1.9 & 1.0 & 1.2 & 0.0 & 0.0 & 0.0 & 0.0 & 0.6 & 0.9 & 0.3 & 0.0 & 0.2 & 0.6 & 0.5 & 0.7 & 0.6 & 36 \\
\hline $17 \mathrm{~F}$ & 0.0 & 2.7 & 0.0 & 1.0 & 0.0 & 0.0 & 0.0 & 0.0 & 0.0 & 0.0 & 2.7 & 0.5 & 0.7 & 1.0 & 0.5 & 0.4 & 0.2 & 0.5 & 31 \\
\hline NT & 0.0 & 0.0 & 0.0 & 0.0 & 1.2 & 0.0 & 0.0 & 0.0 & 0.0 & 0.6 & 0.4 & 0.0 & 0.0 & 0.5 & 0.5 & 0.7 & 0.5 & 0.5 & 30 \\
\hline $15 \mathrm{~A}$ & 0.0 & 0.0 & 0.0 & 2.0 & 0.0 & 0.0 & 3.2 & 1.9 & 0.0 & 1.2 & 1.3 & 0.3 & 0.7 & 0.5 & 0.3 & 0.1 & 0.4 & 0.4 & 27 \\
\hline $15 \mathrm{C}$ & 1.6 & 0.0 & 0.0 & 2.0 & 0.0 & 1.9 & 0.0 & 0.0 & 1.9 & 0.0 & 0.4 & 0.5 & 0.4 & 0.2 & 0.6 & 0.5 & 0.1 & 0.4 & 27 \\
\hline $16 \mathrm{~F}$ & 0.0 & 0.0 & 0.0 & 2.0 & 1.2 & 0.0 & 0.0 & 1.9 & 0.0 & 1.2 & 0.9 & 0.8 & 0.7 & 0.2 & 0.6 & 0.1 & 0.4 & 0.4 & 27 \\
\hline $9 \mathrm{~A}$ & 0.5 & 1.4 & 1.9 & 1.0 & 2.4 & 0.0 & 1.6 & 0.0 & 0.0 & 3.0 & 2.2 & 0.5 & 0.0 & 0.7 & 0.0 & 0.2 & 0.0 & 0.4 & 26 \\
\hline 31 & 1.0 & 0.0 & 1.9 & 1.0 & 0.0 & 0.0 & 0.0 & 0.0 & 0.0 & 1.2 & 0.9 & 0.8 & 0.0 & 0.2 & 0.5 & 0.1 & 0.5 & 0.4 & 25 \\
\hline
\end{tabular}


(c) Continued.

\begin{tabular}{|c|c|c|c|c|c|c|c|c|c|c|c|c|c|c|c|c|c|c|c|}
\hline $\begin{array}{l}\text { Sero- } \\
\text { type }\end{array}$ & $\begin{array}{c}1992 \\
(\%)\end{array}$ & $\begin{array}{c}1993 \\
(\%)\end{array}$ & $\begin{array}{c}1994 \\
(\%)\end{array}$ & $\begin{array}{c}1995 \\
(\%)\end{array}$ & $\begin{array}{c}1996 \\
(\%)\end{array}$ & $\begin{array}{l}1997 \\
(\%)\end{array}$ & $\begin{array}{c}1998 \\
(\%)\end{array}$ & $\begin{array}{c}1999 \\
(\%)\end{array}$ & $\begin{array}{c}2000 \\
(\%) \\
\end{array}$ & $\begin{array}{c}2001 \\
(\%) \\
\end{array}$ & $\begin{array}{c}2002 \\
(\%)\end{array}$ & $\begin{array}{c}2003 \\
(\%) \\
\end{array}$ & $\begin{array}{c}2004 \\
(\%) \\
\end{array}$ & $\begin{array}{c}2005 \\
(\%) \\
\end{array}$ & $\begin{array}{c}2006 \\
(\%) \\
\end{array}$ & $\begin{array}{c}2007 \\
(\%) \\
\end{array}$ & $\begin{array}{c}2008 \\
(\%) \\
\end{array}$ & $\begin{array}{c}\text { Total } \\
(\%)\end{array}$ & $\begin{array}{c}\text { Total } \\
(n)\end{array}$ \\
\hline $33 \mathrm{~A}$ & 0.0 & 0.0 & 0.0 & 0.0 & 0.0 & 0.0 & 0.0 & 0.0 & 0.0 & 0.0 & 0.0 & 0.0 & 0.4 & 0.5 & 0.8 & 0.5 & 0.2 & 0.3 & 21 \\
\hline 34 & 0.5 & 0.0 & 0.0 & 0.0 & 0.0 & 0.0 & 0.0 & 0.0 & 0.0 & 0.0 & 0.9 & 0.5 & 0.0 & 0.2 & 0.3 & 0.2 & 0.2 & 0.2 & 16 \\
\hline 13 & 0.0 & 4.1 & 0.0 & 0.0 & 0.0 & 0.0 & 0.0 & 0.0 & 0.0 & 0.6 & 1.3 & 0.0 & 0.4 & 0.0 & 0.0 & 0.1 & 0.2 & 0.2 & 12 \\
\hline $18 \mathrm{~A}$ & 0.0 & 0.0 & 1.9 & 0.0 & 0.0 & 0.0 & 0.0 & 0.0 & 0.0 & 0.0 & 0.0 & 0.5 & 0.0 & 0.3 & 0.2 & 0.2 & 0.1 & 0.2 & 12 \\
\hline 2 & 0.5 & 0.0 & 1.9 & 1.0 & 0.0 & 1.9 & 0.0 & 1.9 & 0.0 & 0.0 & 0.0 & 0.0 & 0.0 & 0.0 & 0.0 & 0.4 & 0.0 & 0.2 & 12 \\
\hline 9L & 0.0 & 0.0 & 0.0 & 0.0 & 0.0 & 0.0 & 1.6 & 0.0 & 0.0 & 1.2 & 0.9 & 0.3 & 0.4 & 0.0 & 0.2 & 0.2 & 0.0 & 0.2 & 12 \\
\hline $23 \mathrm{~B}$ & 0.0 & 0.0 & 0.0 & 0.0 & 0.0 & 0.0 & 0.0 & 0.0 & 0.0 & 1.2 & 0.0 & 0.0 & 0.4 & 0.2 & 0.2 & 0.1 & 0.2 & 0.2 & 11 \\
\hline rough & 0.0 & 0.0 & 1.9 & 1.0 & 1.2 & 0.0 & 0.0 & 0.0 & 0.0 & 0.0 & 1.3 & 1.3 & 0.0 & 0.0 & 0.0 & 0.0 & 0.0 & 0.2 & 11 \\
\hline $10 \mathrm{~B}$ & 0.0 & 0.0 & 0.0 & 0.0 & 0.0 & 0.0 & 1.6 & 0.0 & 0.0 & 0.0 & 0.0 & 0.0 & 0.4 & 0.3 & 0.0 & 0.2 & 0.2 & 0.2 & 10 \\
\hline $18 \mathrm{~F}$ & 0.5 & 2.7 & 0.0 & 1.0 & 0.0 & 0.0 & 0.0 & م0 & 0.0 & 0 & 0.0 & 0.3 & 0 & 0.2 & 0 . & 0.2 & 0.0 & 0. & 10 \\
\hline $12 \mathrm{~A}$ & 0.0 & 0.0 & 0.0 & 0.0 & 0.0 & 0.0 & 0.0 & 0.0 & 0.0 & 0.0 & 0.4 & 0.0 & 0.0 & 0.0 & 0.5 & 0.2 & 0.1 & 0.1 & 9 \\
\hline $28 \mathrm{~A}$ & 0.0 & 0.0 & 0.0 & 0.0 & 0.0 & 0.0 & 0.0 & 0.0 & 0.0 & 0.0 & 0.9 & 0.3 & 0.0 & 0.0 & 0 . & 0.2 & 0.0 & 0.1 & 8 \\
\hline $35 B$ & 0.0 & 0.0 & 0.0 & 0.0 & 0.0 & 0.0 & 0.0 & 0.0 & 0.0 & 0.0 & 0.0 & 0.0 & 0.0 & 0.0 & 0.0 & 0.2 & 0.2 & 0.1 & 8 \\
\hline $15 \mathrm{~F}$ & 0.5 & 0.0 & 0.0 & 1.0 & 0.0 & 0.0 & 0.0 & 1.9 & 0.0 & 0.0 & 0.4 & 0.3 & 0.4 & 0.0 & 0.0 & 0.1 & 0.0 & 0.1 & 7 \\
\hline $7 \mathrm{C}$ & 0.5 & 0.0 & 0.0 & 1.0 & 0.0 & 0.0 & 0.0 & 0.0 & 0.0 & 0. & 0.0 & 0 . & 0. & 0.2 & 0 . & 0.0 & 0 & 0 . & 7 \\
\hline $11 \mathrm{~F}$ & 0.5 & 0.0 & 0.0 & 0.0 & 0.0 & 0.0 & 0.0 & 0.0 & 0.0 & 0.0 & 0.0 & 0.0 & 0.0 & 0.0 & 0.0 & 0.3 & 0.0 & 0.1 & 6 \\
\hline $10 \mathrm{~F}$ & 0.5 & 0.0 & 0.0 & 0.0 & 0.0 & 1.9 & 0.0 & 0.0 & 0.0 & 0.0 & 0.0 & 0.3 & 0.0 & 0.0 & 0.0 & 0.1 & 0.1 & 0.1 & 5 \\
\hline $12 \mathrm{~B}$ & 0.0 & 0.0 & 0.0 & 0.0 & 0.0 & 0.0 & 0.0 & 0.0 & 0.0 & 0.6 & 0.0 & 0.0 & 0.0 & 0.0 & 0.0 & 0.2 & 0.0 & 0.1 & 5 \\
\hline Others ^ & 1.0 & 2.7 & 1.9 & 6.0 & 1.2 & 0.0 & 8.1 & 0.0 & 0.0 & 1.8 & 0.4 & 1.6 & 0.0 & 0.9 & 0.3 & 0.8 & 0.6 & 0.9 & 58 \\
\hline Total & 100 & 100 & 100 & 100 & 100 & 100 & 100 & 100 & 100 & 100 & 100 & 100 & 100 & 100 & 100 & 100 & 100 & 100 & 6,446 \\
\hline $\begin{array}{l}\text { Not } \\
\text { sero- } \\
\text { typed }\end{array}$ & 57.5 & 82.8 & 82.8 & 69.6 & 68.4 & 71.3 & 70.5 & 77.1 & 73.4 & 61.4 & 47.7 & 30.7 & 31.8 & 2.3 & 0.0 & 0.1 & 0.0 & 29.3 & 2,671 \\
\hline
\end{tabular}

NT: nontypeable; $n$ : number of isolates tested.

Others $^{\star}$ includes the serotypes (number of isolates): 33B (4), 35A (4), 11B (3), 19C (3), 22A (3), 29 (3), 35C (3), 37 (3), 45 (3), 18B (2), 19B (2), 21 (2), 24A (2), 24B (2), 25F (2), 28F (2), 36 (2), 48 (2), 7B (2), 12 (1), 17A (1), 18 (1), 19 (1), 23 (1), 39 (1), 6 (1), 7A (1), 9 (1).

TABLE 2: Distribution of isolates serotyped among pneumococcal isolates sent to the NRCS in Germany (1992-2008).

\begin{tabular}{|c|c|c|c|c|c|c|c|c|c|c|c|c|c|c|c|c|c|}
\hline $\begin{array}{l}\text { Isolates } \\
\text { serotyped }\end{array}$ & $\begin{array}{c}1992 \\
(\%)\end{array}$ & $\begin{array}{l}1993 \\
(\%) \\
\end{array}$ & $\begin{array}{l}1994 \\
(\%)\end{array}$ & $\begin{array}{l}1995 \\
(\%)\end{array}$ & $\begin{array}{l}1996 \\
(\%)\end{array}$ & $\begin{array}{l}1997 \\
(\%)\end{array}$ & $\begin{array}{l}1998 \\
(\%)\end{array}$ & $\begin{array}{l}1999 \\
(\%)\end{array}$ & $\begin{array}{c}2000 \\
(\%)\end{array}$ & $\begin{array}{c}2001 \\
(\%) \\
\end{array}$ & $\begin{array}{c}2002 \\
(\%)\end{array}$ & $\begin{array}{c}2003 \\
(\%) \\
\end{array}$ & $\begin{array}{c}2004 \\
(\%)\end{array}$ & $\begin{array}{c}2005 \\
(\%)\end{array}$ & $\begin{array}{c}2006 \\
(\%)\end{array}$ & $\begin{array}{c}2007 \\
(\%) \\
\end{array}$ & $\begin{array}{c}2008 \\
(\%) \\
\end{array}$ \\
\hline \multicolumn{18}{|l|}{ children } \\
\hline $\begin{array}{l}\text { serotyped } \\
(\%)\end{array}$ & 92,2 & 82,9 & 96,6 & 65,4 & 38,7 & 92,9 & 97,6 & 96,8 & 96,2 & 100,0 & 99,6 & 99,2 & 100,0 & 100,0 & 100,0 & 100,0 & 100,0 \\
\hline $\begin{array}{l}\text { serotyped } \\
(n)\end{array}$ & 47 & 29 & 28 & 17 & 12 & 158 & 161 & 182 & 200 & 239 & 258 & 244 & 265 & 316 & 287 & 273 & 232 \\
\hline total & 51 & 35 & 29 & 26 & 31 & 170 & 165 & 188 & 208 & 239 & 259 & 246 & 265 & 316 & 287 & 273 & 232 \\
\hline \multicolumn{18}{|l|}{ adults } \\
\hline $\begin{array}{l}\text { serotyped } \\
(\%)\end{array}$ & 42,5 & 17,2 & 17,2 & 30,4 & 31,6 & 28,7 & 29,5 & 22,9 & 26,6 & 38,6 & 52,3 & 69,3 & 68,2 & 97,7 & 100,0 & 99,9 & 100,0 \\
\hline $\begin{array}{l}\text { serotyped } \\
(n)\end{array}$ & 193 & 74 & 53 & 100 & 84 & 54 & 62 & 53 & 54 & 165 & 225 & 384 & 270 & 588 & 628 & 1654 & 1805 \\
\hline Total & 454 & 429 & 309 & 329 & 266 & 188 & 210 & 231 & 203 & 428 & 430 & 554 & 396 & 602 & 628 & 1655 & 1805 \\
\hline \multicolumn{18}{|l|}{ overall } \\
\hline $\begin{array}{l}\text { serotyped } \\
(\%)\end{array}$ & 47,5 & 22,2 & 24,0 & 33,0 & 32,3 & 59,2 & 59,5 & 56,1 & 61,8 & 60,6 & 70,1 & 78,5 & 80,9 & 98,5 & 100,0 & 99,9 & 100,0 \\
\hline $\begin{array}{l}\text { serotyped } \\
(n)\end{array}$ & 240 & 103 & 81 & 117 & 96 & 212 & 223 & 235 & 254 & 404 & 483 & 628 & 535 & 904 & 915 & 1927 & 2037 \\
\hline total & 505 & 464 & 338 & 355 & 297 & 358 & 375 & 419 & 411 & 667 & 689 & 800 & 661 & 918 & 915 & 1928 & 2037 \\
\hline
\end{tabular}




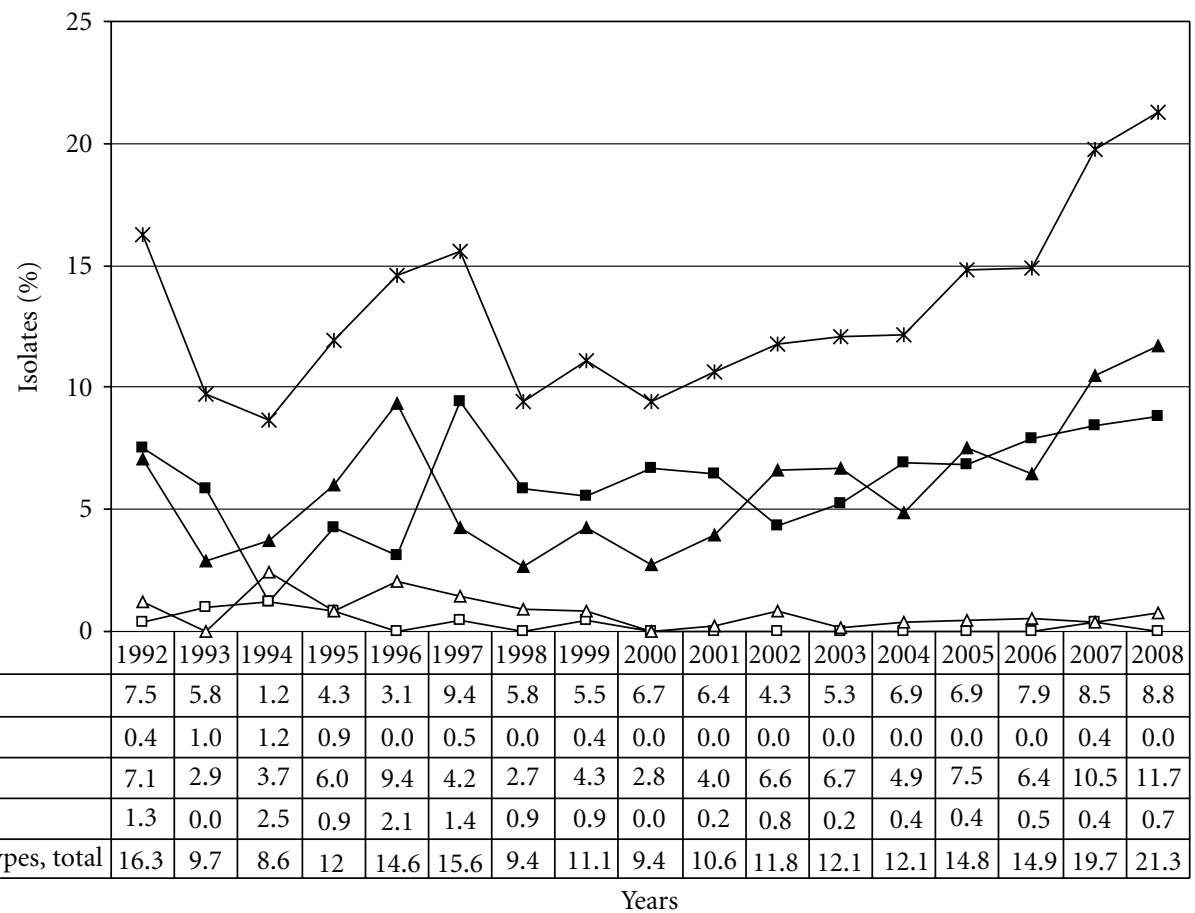

(a)

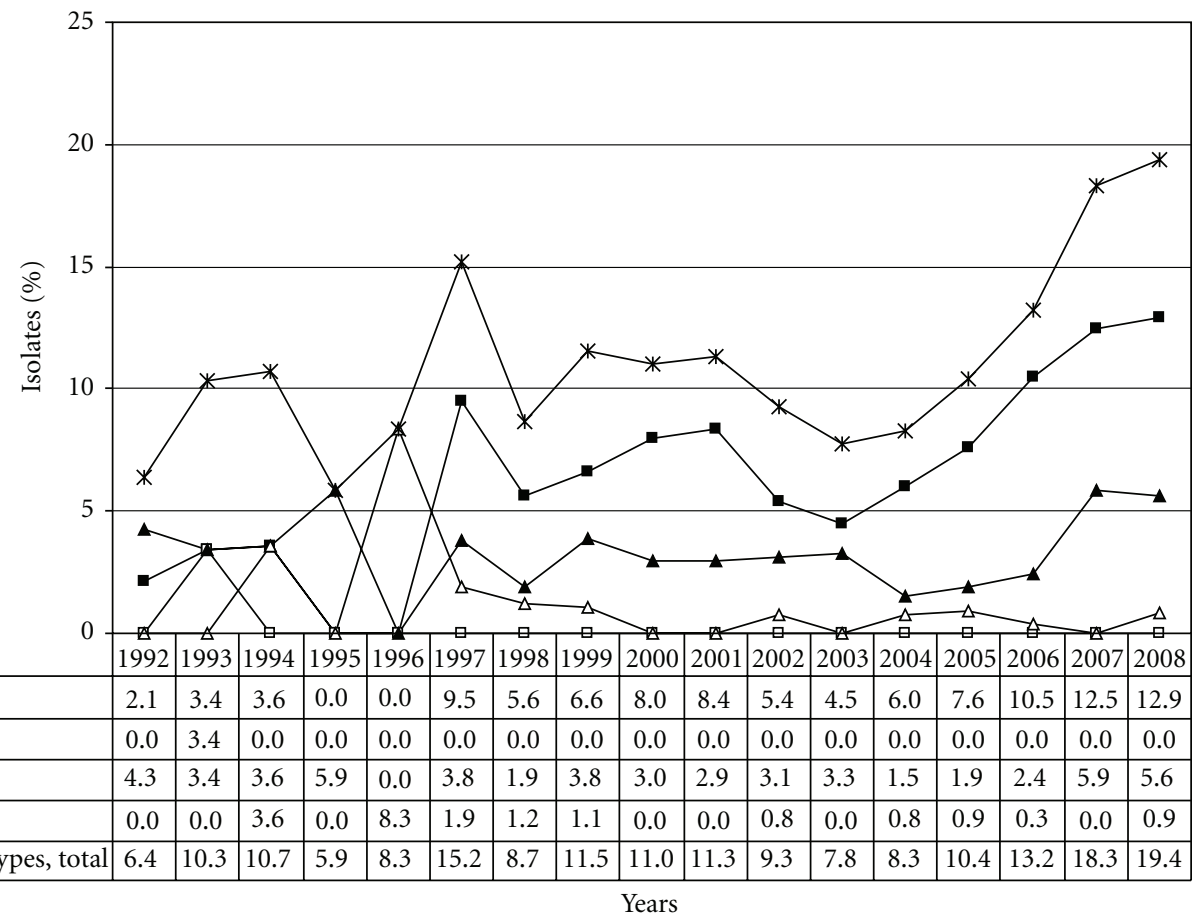

(b)

Figure 1: Continued. 


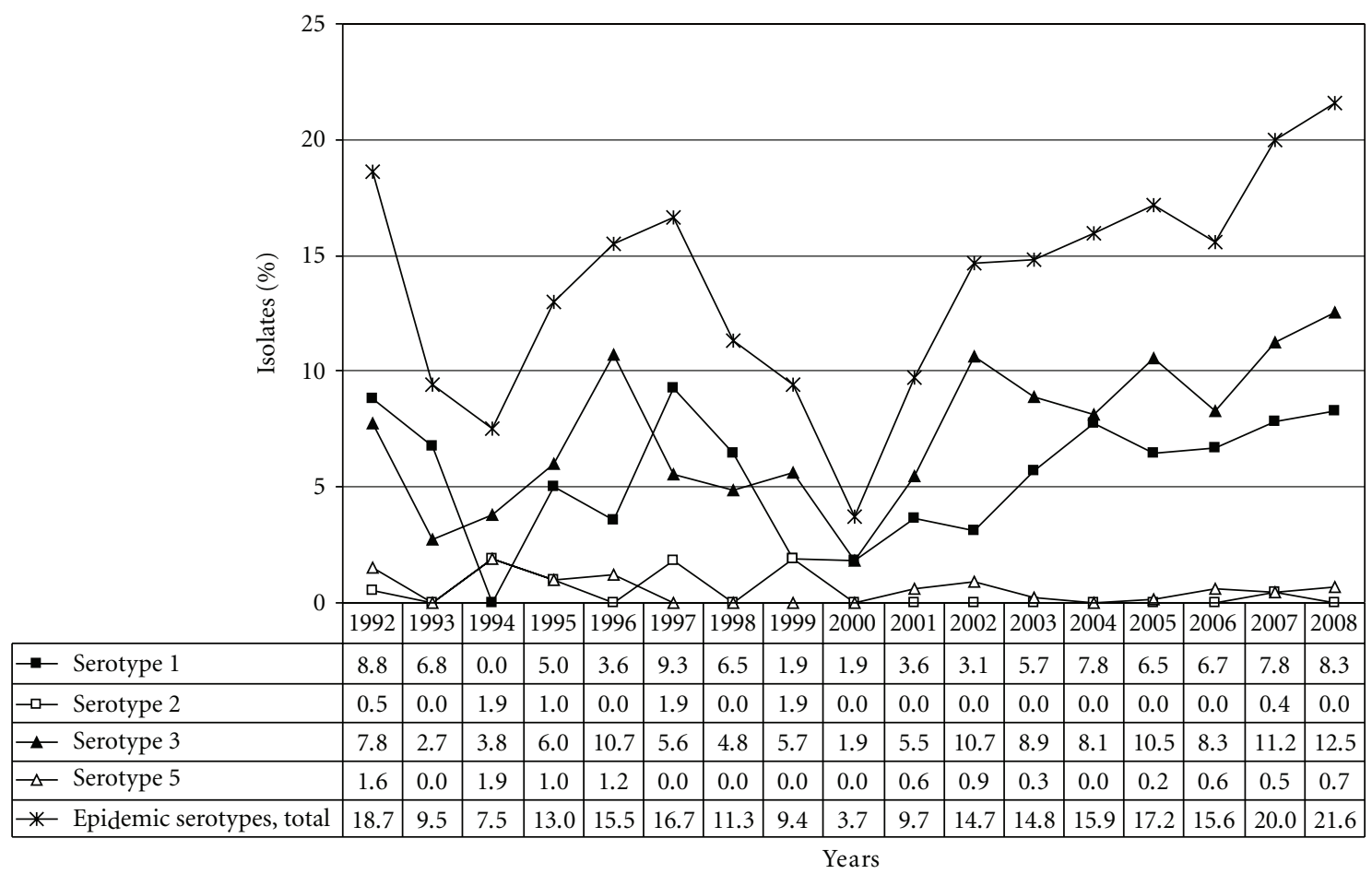

(c)

Figure 1: (a) Distribution of the epidemic serotypes 1, 2, 3, and 5 in percent $(1992-2008, n=9,394)$ in all age groups. (b) Distribution of the epidemic serotypes $1-3$ and 5 among children in percent (1992-2008, $n=2,948$ ). (c) Distribution of the epidemic serotypes $1-3$ and 5 among adults in percent $(1992-2008, n=6,446)$.

vaccine coverages for the 7 -valent, 10 -valent, and 13 -valent pneumococcal conjugate vaccines.

\section{Materials and Methods}

2.1. Study Design. In this study data about invasive disease caused by Streptococcus pneumoniae in children ( $<16$ years) and adults ( $\geq 16$ years) in Germany were included using the data sources described above. Cases from January 1, 1992 to December 31, 2008 were included in this study. A case of IPD was defined by the isolation of S. pneumoniae from a normally sterile site.

2.2. Microbiological Investigations. Isolates were identified using standard procedures including bile solubility and optochin sensitivity. As a control strain, Streptococcus pneumoniae ATCC 49619 was used. Pneumococcal isolates were serotyped by the Neufeld's Quellung reaction using type- and factor-specific antisera (Statens Serum Institut, Copenhagen, Denmark). Among isolates of adults, high levels of resistance were a main trigger for initiation of serotyping during the early years of this study. Since cross-reactive serotypes were not included in the calculation, coverage information strictly refers to the serotypes included in the vaccines. Serogroup 6 isolates were not tested with respect to serotype 6C.

\section{Results}

A total of 12,137 isolates from invasive pneumococcal disease were collected between January 1, 1992 and December 31,
2008. The total numbers of cases for each year varied between 297 and 2,037 cases (median: 505 cases). Data on serotypes were available for 9,394 isolates $(77.4 \%$ of all invasive isolates; $31.4 \%$ children, $68.6 \%$ adults; $54.9 \%$ male, $43.7 \%$ female, $1.4 \%$ no information on gender). The serotype distribution of the years 1992-2008 is shown in Table 1(a). The leading serotypes were serotypes $14(16.5 \%)$, $3(8.0 \%), 7 \mathrm{~F}(7.6 \%), 1$ (7.3\%), and 23F (6.0\%). Concerning the epidemic serotypes $1-3$ and 5, a slight increase was noticed during the last years, reaching levels around 20\% among children and adults in 2008 (Figures 1(a)-1(c)). Variations in serotype distribution over the years are partly extensive, especially concerning serotype 14 ( $\min 7.4 \%$, max $33.5 \%$ ) (Figure 2(a)). Differences in serotype distribution among children and adults are shown in Tables 1(b) and 1(c). The amount of serotyped isolates among all pneumococcal isolates sent to the NRCS in Germany is shown in Table 2. Over the years the percentage of isolates serotyped increased continuously and in the last four years of the study almost all isolates were serotyped.

Serotype 14 is considerably more frequent among children $(22.5 \%)$ than among adults (13.7\%). Overall, an increase of serotype 14 can be noticed from around 1997 to 2006 (Figure 2(a)). A high percentage of serotype 14 isolates remain in existence for a longer time among children (1999-2006, Figure 2(b)) than among adults (1999-2001, Figure 2(c)). Serotype 1 and serotype $7 \mathrm{~F}$ have been clearly increasing over the last approximately 10 years, both among children and among adults. While for children the percentage 


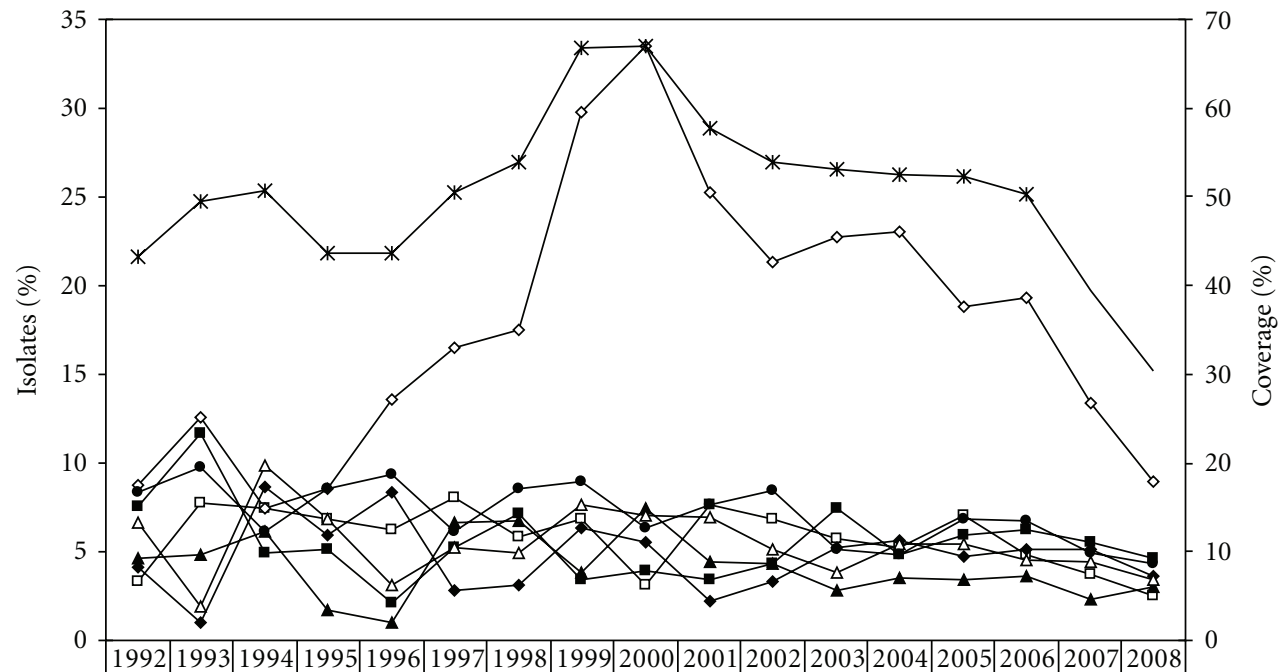

\begin{tabular}{|l|c|c|c|c|c|c|c|c|c|c|c|c|c|c|c|c|c|}
\hline$\rightarrow-4$ & 7.5 & 11.7 & 4.9 & 5.1 & 2.1 & 5.2 & 7.2 & 3.4 & 3.9 & 3.5 & 4.3 & 7.5 & 4.9 & 6.0 & 6.2 & 5.6 & 4.6 \\
\hline$\square-6 \mathrm{~B}$ & 3.3 & 7.8 & 7.4 & 6.8 & 6.3 & 8.0 & 5.8 & 6.8 & 3.1 & 7.7 & 6.8 & 5.7 & 5.2 & 7.1 & 4.8 & 3.7 & 2.5 \\
\hline$\multimap-9 \mathrm{~V}$ & 4.2 & 1.0 & 8.6 & 6.0 & 8.3 & 2.8 & 3.1 & 6.4 & 5.5 & 2.2 & 3.3 & 5.3 & 5.6 & 4.8 & 5.1 & 5.1 & 3.6 \\
\hline$\multimap-14$ & 8.8 & 12.6 & 7.4 & 8.5 & 13.5 & 16.5 & 17.5 & 29.8 & 33.5 & 25.2 & 21.3 & 22.8 & 23.0 & 18.8 & 19.3 & 13.3 & 8.9 \\
\hline$\multimap 18 \mathrm{C}$ & 4.6 & 4.9 & 6.2 & 1.7 & 1.0 & 6.6 & 6.7 & 3.8 & 7.5 & 4.5 & 4.3 & 2.9 & 3.6 & 3.4 & 3.6 & 2.3 & 3.0 \\
\hline$\rightarrow-19 \mathrm{~F}$ & 6.7 & 1.9 & 9.9 & 6.8 & 3.1 & 5.2 & 4.9 & 7.7 & 7.1 & 6.9 & 5.2 & 3.8 & 5.4 & 5.4 & 4.5 & 4.5 & 3.4 \\
\hline$\bullet-23 \mathrm{~F}$ & 8.3 & 9.7 & 6.2 & 8.5 & 9.4 & 6.1 & 8.5 & 8.9 & 6.3 & 7.7 & 8.5 & 5.1 & 4.9 & 6.9 & 6.8 & 4.9 & 4.3 \\
\hline$\rightarrow-$ Coverage 7v & 43.3 & 49.5 & 50.6 & 43.6 & 43.8 & 50.5 & 53.8 & 66.8 & 66.9 & 57.7 & 53.8 & 53.0 & 52.5 & 52.3 & 50.4 & 39.3 & 30.4 \\
\hline
\end{tabular}

Years

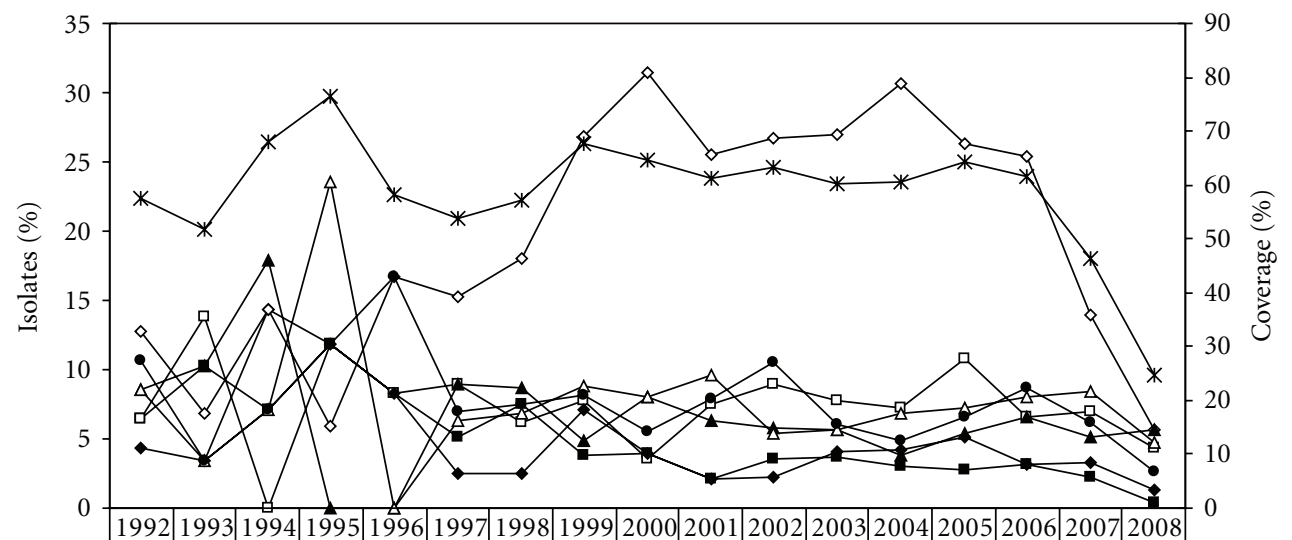

\begin{tabular}{|c|c|c|c|c|c|c|c|c|c|c|c|c|c|c|c|c|c|}
\hline$\rightarrow-4$ & 6.4 & 10.3 & 7.1 & 11.8 & 8.3 & 5.1 & 7.5 & 3.8 & 4.0 & 2.1 & 3.5 & 3.7 & 3.0 & 2.8 & 3.1 & 2.2 & 0.4 \\
\hline$-\square-6 \mathrm{~B}$ & 6.4 & 13.8 & 0.0 & 11.8 & 8.3 & 8.9 & 6.2 & 7.7 & 3.5 & 7.5 & 8.9 & 7.8 & 7.2 & 10.8 & 6.6 & 7.0 & 4.3 \\
\hline$\multimap-9 \mathrm{~V}$ & 4.3 & 3.4 & 14.3 & 11.8 & 8.3 & 2.5 & 2.5 & 7.1 & 4.0 & 2.1 & 2.3 & 4.1 & 4.2 & 5.1 & 3.1 & 3.3 & 1.3 \\
\hline$\multimap \quad 14$ & 12.8 & 6.9 & 14.3 & 5.9 & 16.7 & 15.2 & 18.0 & 26.9 & 31.5 & 25.5 & 26.7 & 27.0 & 30.6 & 26.3 & 25.4 & 13.9 & 5.6 \\
\hline$\rightarrow 18 \mathrm{C}$ & 8.5 & 10.3 & 17.9 & 0.0 & 0.0 & 8.9 & 8.7 & 4.9 & 8.0 & 6.3 & 5.8 & 5.7 & 3.8 & 5.4 & 6.6 & 5.1 & 5.6 \\
\hline$-\Delta-19 \mathrm{~F}$ & 8.5 & 3.4 & 7.1 & 23.5 & 0.0 & 6.3 & 6.8 & 8.8 & 8.0 & 9.6 & 5.4 & 5.7 & 6.8 & 7.3 & 8.0 & 8.4 & 4.7 \\
\hline$\rightarrow 23 \mathrm{~F}$ & 10.6 & 3.4 & 7.1 & 11.8 & 16.7 & 7.0 & 7.5 & 8.2 & 5.5 & 7.9 & 10.5 & 6.1 & 4.9 & 6.6 & 8.7 & 6.2 & 2.6 \\
\hline$*$ Coverage $7 \mathrm{v}$ & 57.4 & 51.7 & 67.9 & 76.5 & 58.3 & 53.8 & 57.1 & 67.6 & 64.5 & 61.1 & 63.2 & 60.2 & 60.4 & 64.2 & 61.7 & 46.2 & 24.6 \\
\hline
\end{tabular}

Years

(b)

Figure 2: Continued. 


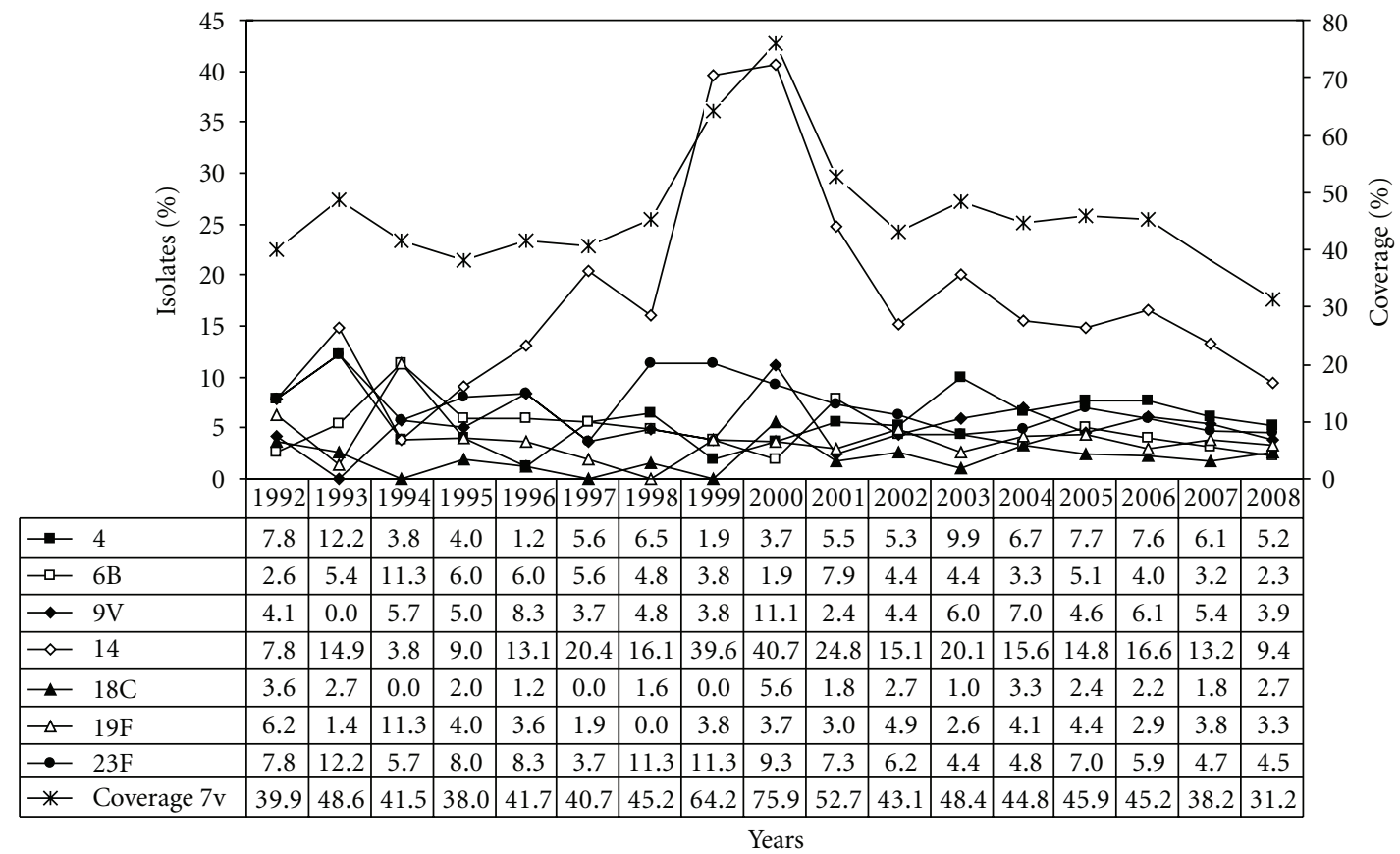

(c)

Figure 2: (a) Distribution of the 7-valent pneumococcal conjugate vaccine serotypes and the 7-valent vaccine coverage in percent (19922008, $n=9,394$ ). (b) Distribution of the 7 -valent pneumococcal conjugate vaccine serotypes and the 7 -valent vaccine coverage among children in percent (1992-2008, $n=2$,948). (c) Distribution of the 7-valent pneumococcal conjugate vaccine serotypes and the 7-valent vaccine coverage among adults in percent $(1992-2008, n=6,446)$.

of serotypes 1 and $7 \mathrm{~F}$ reach the highest values during this study in 2008, among adults they are high, but still within the range already noted before (Figures 3(a)-3(c)). Serotype 3 is far more common among adults $(10.2 \%)$ than among children (3.3\%) (Tables 1(b)-1(c)). The increase observed during the last years seems to be slightly higher among adults than among children (Figures 4(a)-4(c)). Concerning serotype 19A no clear change in frequency can be observed during the period under study (Figures 4(a)-4(c)).

Variations in serotype distribution also affect theoretical vaccine coverages. The overall serotype coverage for the 7valent conjugate vaccine was $45.4 \%$ during $1992-2008$. For the 10 -valent vaccine and the 13 -valent vaccine the overall coverages were $60.9 \%$ and $76.6 \%$, respectively. Generally, higher coverages were observed among children $(7 \mathrm{v}, 57.3 \%$; $10 \mathrm{v}, 72.8 \% ; 13 \mathrm{v}, 83.5 \%)$ than among adults $(7 \mathrm{v}, 39.9 \% ; 10 \mathrm{v}$, $55.5 \% ; 13 \mathrm{v}, 73.5 \%)$. Among children, the coverage of the $7-$ valent vaccine decreased from $64.2 \%$ in 2005 to $24.6 \%$ in 2008 (Figure 2(b)), while for adults the coverage declined to a lesser extent $(2005,45.9 \% ; 2008,31.2 \%)$ (Figure 2(c)). Temporal changes of the additional 10 -valent and 13 -valent pneumococcal vaccine serotypes and the corresponding vaccine coverages are shown in Figures 3(a)-3(c) (10v) and Figures $4(\mathrm{a})-4(\mathrm{c})(13 \mathrm{v})$. Since the general recommendation of pneumococcal conjugate vaccination for children $<2$ years in Germany at the end of July 2006 a percentage increase among the new $(10 \mathrm{v})$ or upcoming $(13 \mathrm{v})$ vaccine serotypes was noticed especially for serotypes 1 and $7 \mathrm{~F}$ in children (Figure $3(\mathrm{~b})$ ) and for serotype 3 in adults (Figure 4(c)).
In comparison, coverages of the 23-valent polysaccharide vaccine are very similar among children ( $\min , 69.0 \%$; $\max$, 92.3\%) and adults ( $\min , 71.0 \%$; $\max , 92.6 \%$ ) from 1992 to 2008 (Figure 5).

\section{Discussion}

In this paper we present the results of 17 years of surveillance concerning serotypes of invasive pneumococcal disease in Germany.

In the present study serotype 14 was the most prevalent serotype among children, followed in frequency by the serotypes $1,6 \mathrm{~B}, 19 \mathrm{~F}, 23 \mathrm{~F}$, and 7F, respectively. These data are similar to those published for German children previously $[12,13]$ and in line with data from England [14], Belgium [15], and Denmark [16]. Among adults, the most frequent serotypes were $14,3,7 \mathrm{~F}, 1,4$, and $23 \mathrm{~F}$ (sorted in descending order). These results are similar to a previous study from the NRCS among adults in North-Rhine Westphalia, Germany, between 2003 and 2006 [17], and are generally in line with results reported from other countries [6, 15, 18-20]; however, they deviate in part from older German data [12, 21].

The variation of serotype 14 over the years is extensive in our study with an increased prevalence from about 1997 to 2006, reaching maximum values in 2000. Although the rate of serotyped isolates among adults was low from 1999 to 2001 (1999, 22.9\%; 2000, 26.6\%; 2001, 38.6\%), the increase seems plausible. First, the increase among adults is paralleled by an increase among children, where nearly all isolates have 


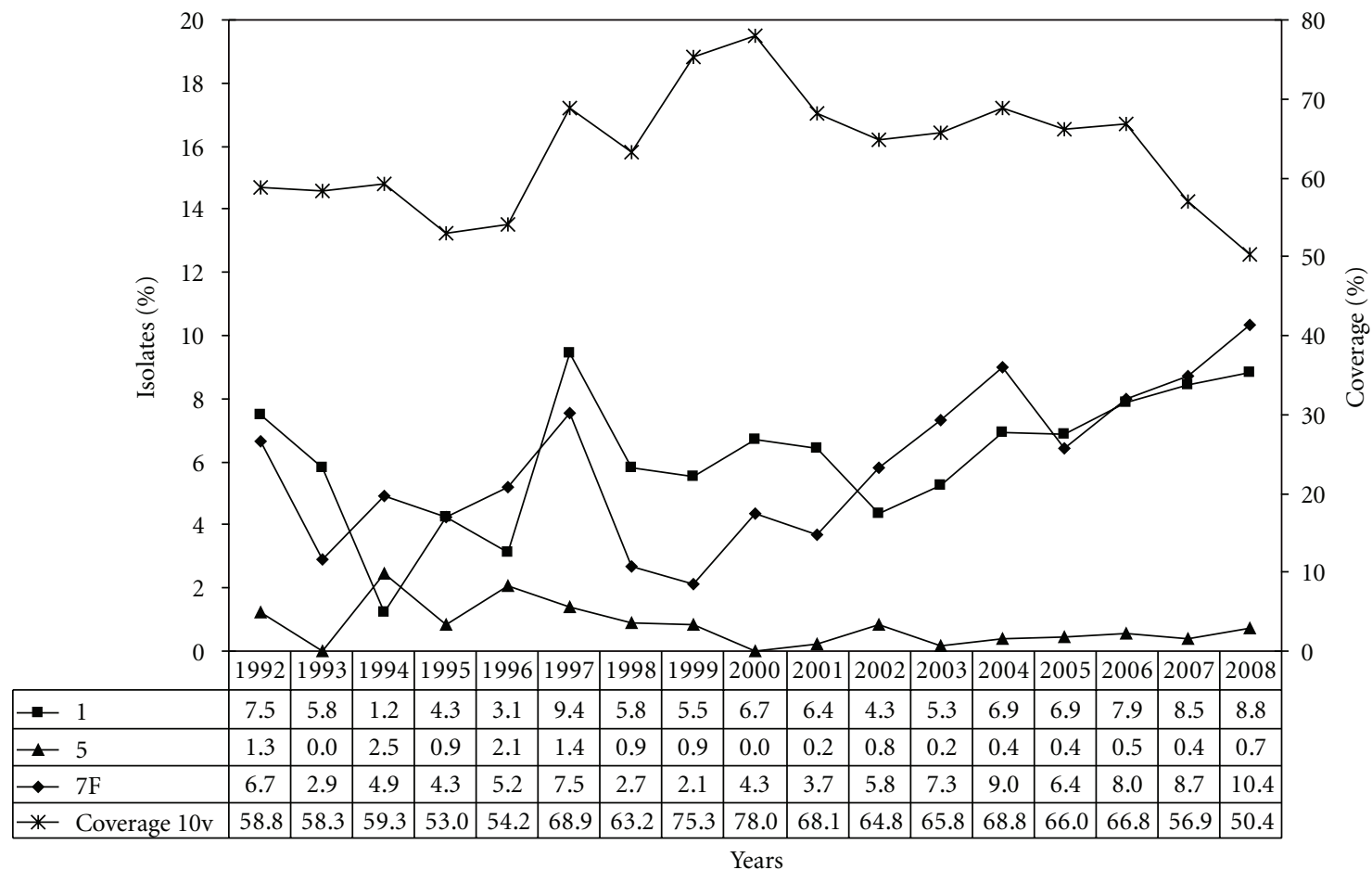

(a)

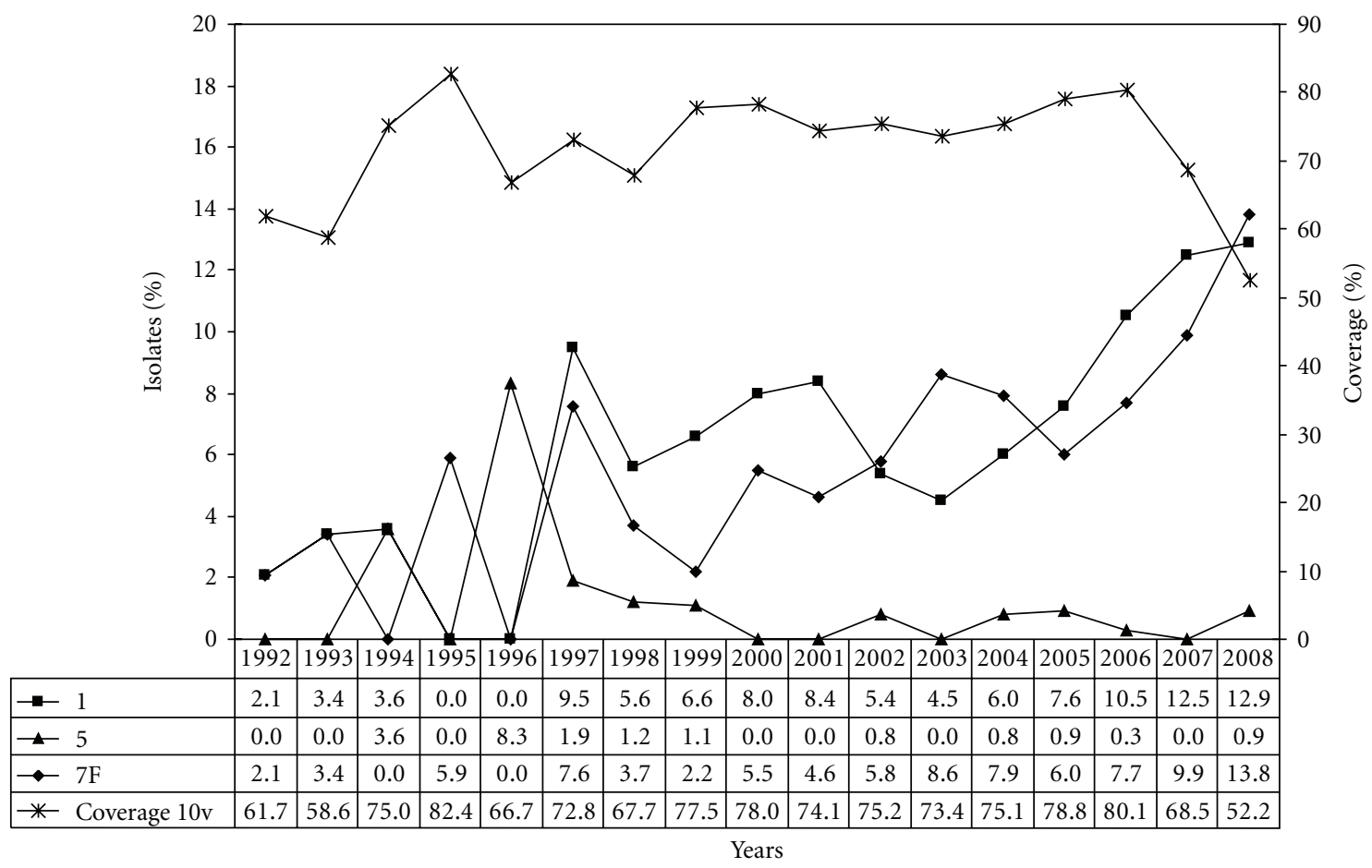

(b)

Figure 3: Continued. 


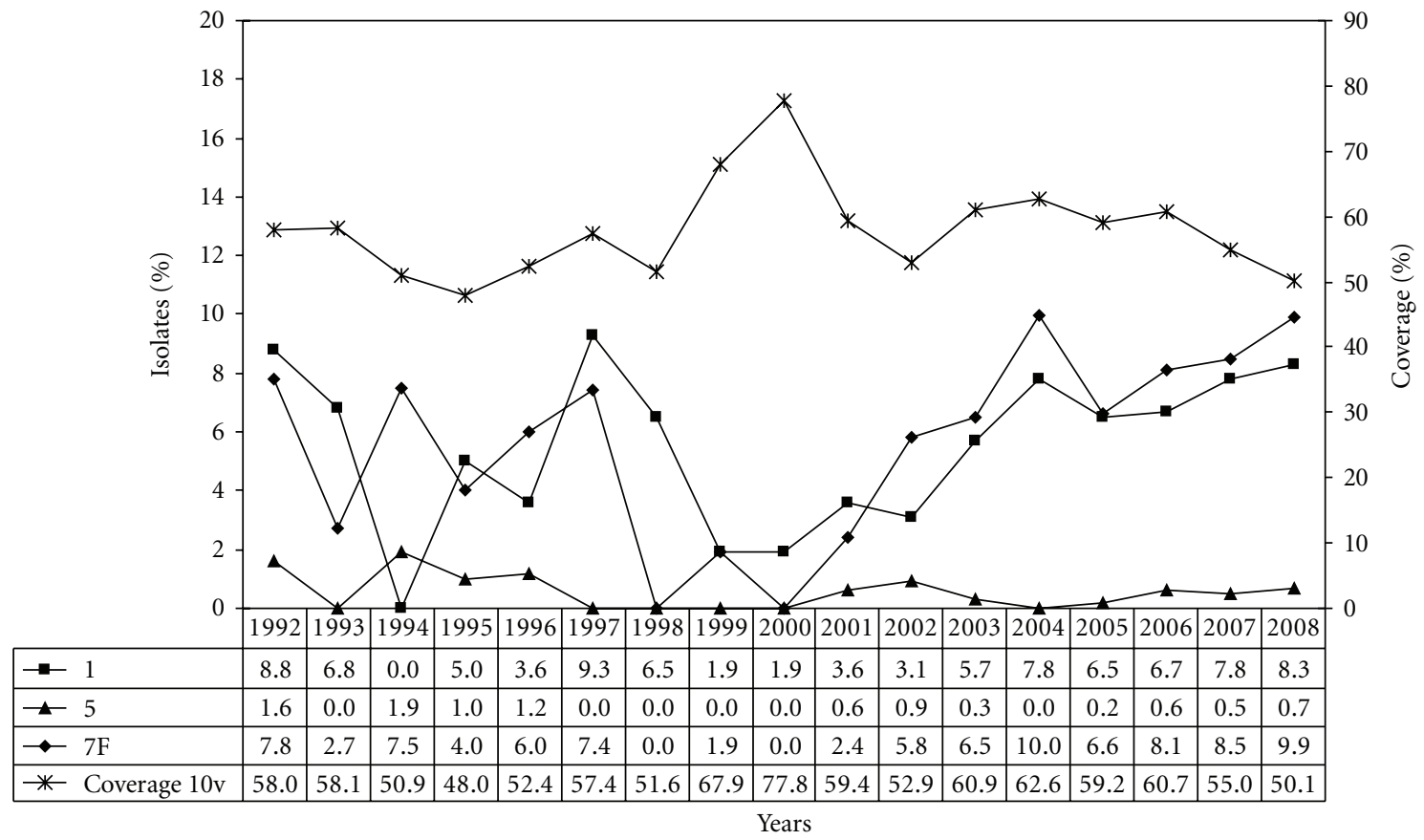

(c)

Figure 3: (a) Distribution of the additional 10v-pneumococcal conjugate vaccine serotypes 1, 5, and 7F and the 10-valent vaccine coverage in percent (1992-2008, $n=9,394)$. (b) Distribution of the additional 10v-pneumococcal conjugate vaccine serotypes 1,5 , and 7F and the 10 -valent vaccine coverage among children in percent (1992-2008, $n=2,948)$. (c) Distribution of the additional 10v-pneumococcal conjugate vaccine serotypes 1,5 , and $7 \mathrm{~F}$ and the 10 -valent vaccine coverage among adults in percent $(1992-2008, n=6,446)$.

been serotyped. Second, the rate of isolates serotyped among adults is within the range serotyped in the years before 1999, where a similar increase of serotype 14 was not observed. Furthermore, similar data have been shown in a report from Spain [4]. Also, a small rise in frequency of serotype 14 has been reported from Denmark in 1995-1999 [16], and data from England demonstrated a rise in incidence in 2000 and 2001 [14]. The rise of serotypes 1 and 7F for nearly one decade is similar to results from Spain [4].

Concerning serotype 19A, the highest prevalences were observed in 1996 (8.3\%) and 1998 (6.2\%) among children, and in 1999 (7.5\%) and 2000 (7.4\%) among adults. Although there seems to be a slight upward movement during the years 2002 to 2008, the prevalences still are below the maximum values reported. The increase of serotype 19A reported from other countries $[4,22,23]$ has not been found in Germany so far.

Although changes in the incidence of different serotypes have been reported, the reasons for this are not fully understood [14]. Potential reasons discussed are changes in socioeconomic conditions, antibiotic use and resistance levels, immunocompromised status of populations, precise age distributions of the populations studied and increased life expectancy, and blood-culturing rates [2, 6, 24]. Since the general recommendation of pneumococcal conjugate vaccination for children $<2$ years in Germany at end of July 2006 a reduction in the percentage of IPD caused by the 7 -valent vaccine serotypes was observed [25]. This effect is more apparent among children, but also present among the adult population. Similar results have been reported from other countries [3, 4, 26-29]. Logically, the percentage decrease in vaccine serotypes is paralleled by a percentage increase in the amount of IPD caused by nonvaccine serotypes, which was most prominent for serotypes 1 and $7 \mathrm{~F}$ in children and serotype 3 in adults in this study. Serotype coverage for the 7 -valent conjugate vaccine in Germany was $45.4 \%$ from 1992 to 2008 . Calculated coverages for the 10 valent vaccine and 13 -valent vaccine are $60.9 \%$ and $76.6 \%$, respectively. These data are comparable to those previously reported $[5,14,30,31]$. Coverages of all three vaccine formulations in our study are approximately $15 \%$ (7v, 17.4\%; $10 \mathrm{v}, 17.3 \%$; and $13 \mathrm{v}, 10.0 \%$ ) higher among children than among adults. Differences in age distribution among study populations are known to have a major impact on vaccine coverage of the current and proposed vaccines for different age groups and, furthermore, comparable data have been published [14].

Nevertheless, some factors and limitations must be regarded when interpreting the results of this study. First of all, the isolates were sent by the participating laboratories on a voluntary basis, as participation in surveillance is not mandatory in Germany. Furthermore, the systematic sampling of invasive isolates from adults (1992) and children (1997) was taken up at different points of time, and the included population-based studies in three German federal states started in 2001 (North Rhine-Wesphalia) and 2006 


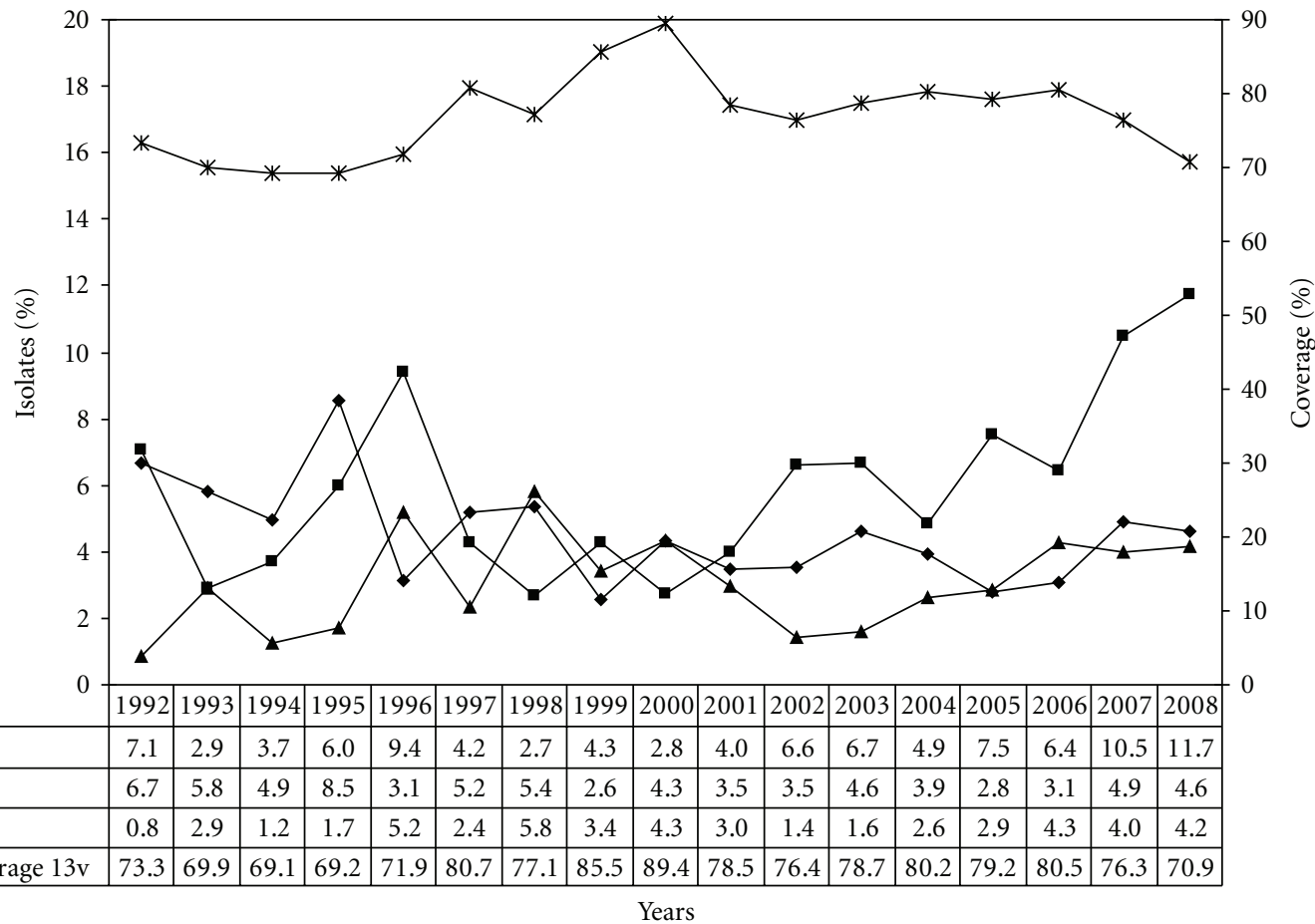

(a)

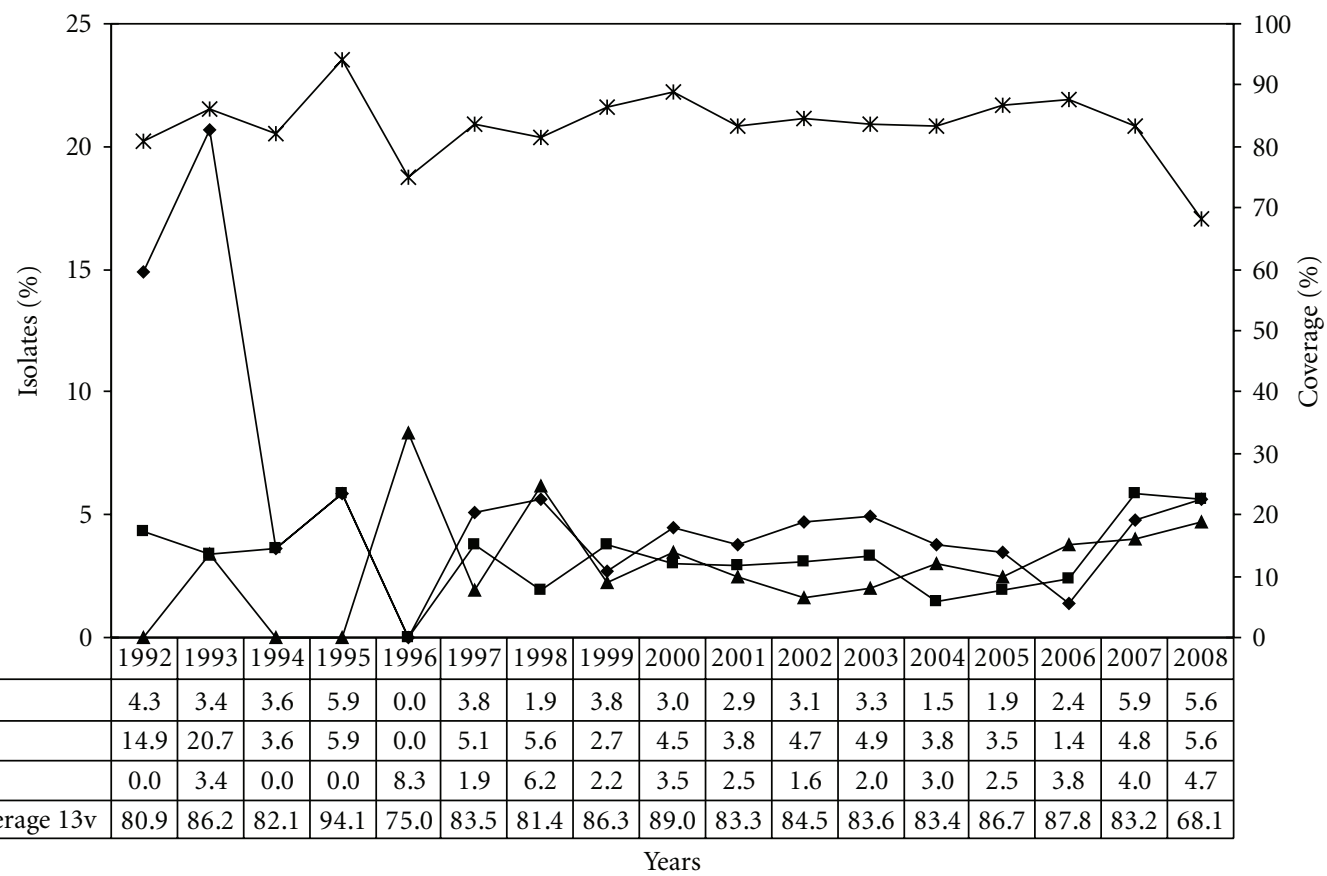

(b)

Figure 4: Continued. 


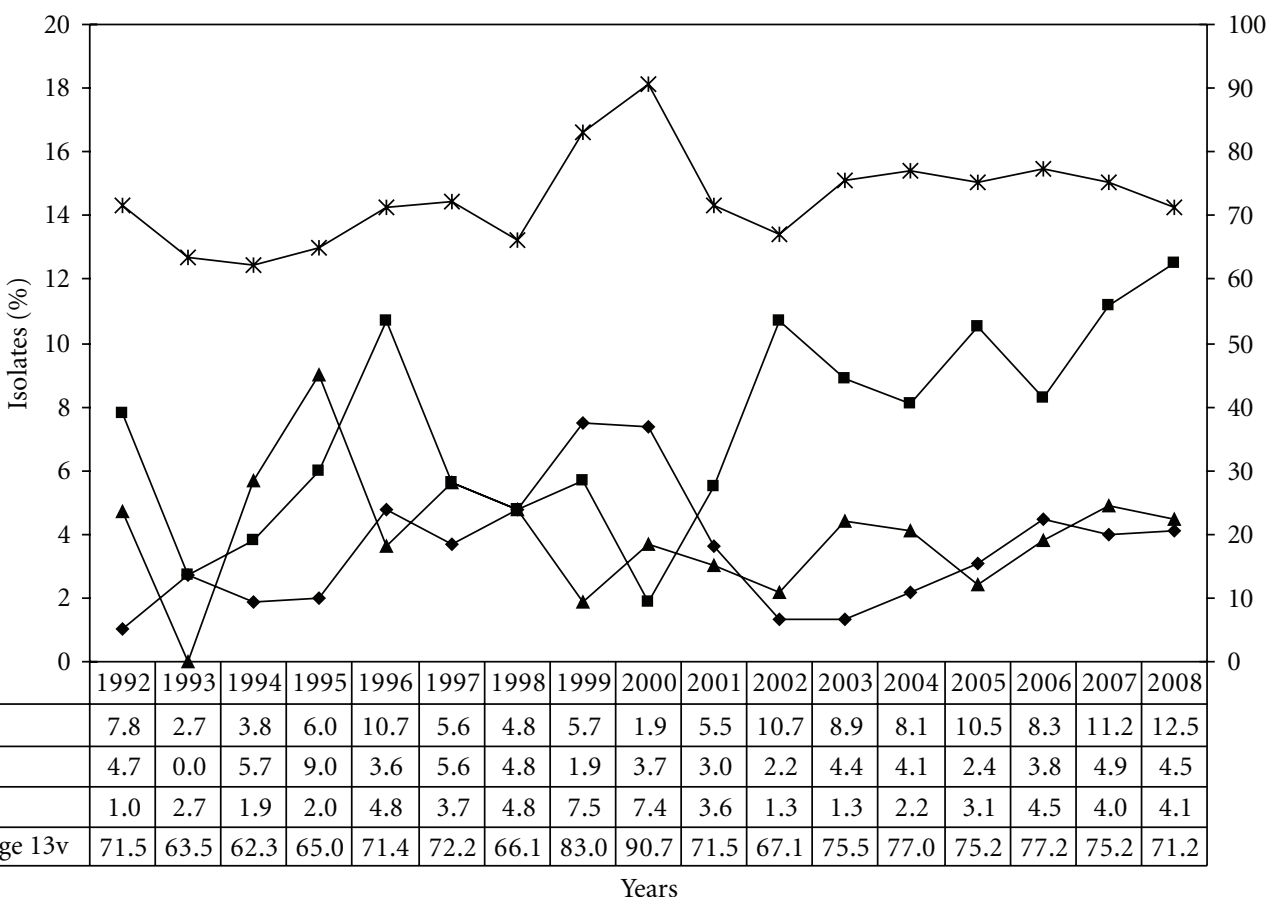

(c)

Figure 4: (a) Distribution of the additional 13v-pneumococcal conjugate vaccine serotypes 3, 6A, and 19A and the 13-valent vaccine coverage in percent $(1992-2008, n=9,394)$. (b) Distribution of the additional 13v-pneumococcal conjugate vaccine serotypes 3, 6A, and 19A and the 13-valent vaccine coverage among children in percent (1992-2008, $n=2,948)$. (c) Distribution of the additional $13 \mathrm{v}$-pneumococcal conjugate vaccine serotypes 3,6A, and 19A and the 13-valent vaccine coverage among adults in percent (1992-2008, $n=6,446)$.

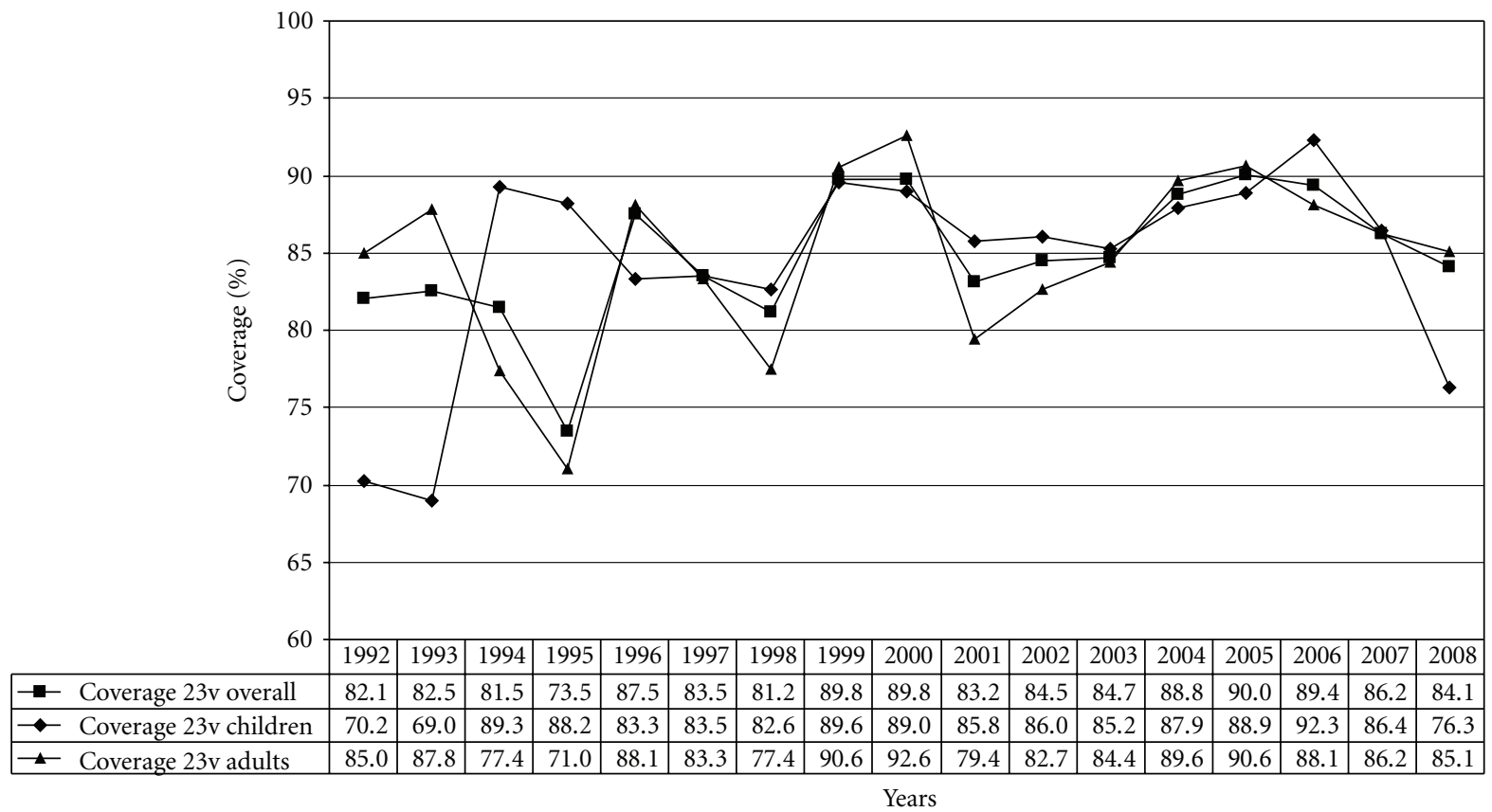

FIGURE 5: Coverage of the 23v-pneumococcal polysaccharide vaccine for children $(n=2,948)$, adults $(n=6,446)$, and overall $(n=9,394)$. 
(Bavaria and Saxony). Among isolates of adults, high levels of resistance were a main trigger for initiation of serotyping during the early years of this study. Therefore, the isolates may not be fully representative of all IPD in Germany over the last two decades.

Moreover, the structure of the surveillance project has been continousely improved over the 15 years and, in particular, after the general recommendation of pneumococcal conjugate vaccine for children $<2$ years in Germany at the end of July 2006 an increased disease awareness by both clinical micobiologists and pediatricians was observed [32].

Ongoing nationwide surveillance is necessary to observe further developments of pneumococcal serotype distribution in Germany.

\section{Acknowledgment}

The authors thank the microbiological laboratories in Germany for their cooperation and for providing the isolates.

\section{References}

[1] R. Austrian, "Pneumococcus: the first one hundred years," Reviews of Infectious Diseases, vol. 3, no. 2, pp. 183-189, 1981.

[2] D. R. Feikin and K. P. Klugman, "Historical changes in pneumococcal serogroup distribution: implications for the era of pneumococcal conjugate vaccines," Clinical Infectious Diseases, vol. 35, no. 5, pp. 547-555, 2002.

[3] M. R. Jacobs, C. E. Good, B. Beall, S. Bajaksouzian, A. R. Windau, and C. G. Whitney, "Changes in serotypes and antimicrobial susceptibility of invasive Streptococcus pneumoniae strains in Cleveland: a quarter century of experience," Journal of Clinical Microbiology, vol. 46, no. 3, pp. 982-990, 2008.

[4] A. Fenoll, J. J. Granizo, L. Aguilar et al., "Temporal trends of invasive Streptococcus pneumoniae serotypes and antimicrobial resistance patterns in Spain from 1979 to 2007," Journal of Clinical Microbiology, vol. 47, no. 4, pp. 1012-1020, 2009.

[5] R. R. Reinert, "Pneumococcal conjugate vaccines-a European perspective," International Journal of Medical Microbiology, vol. 294, no. 5, pp. 277-294, 2004.

[6] D. R. Feikin, K. P. Klugman, R. R. Facklam, E. R. Zell, A. Schuchat, and C. G. Whitney, "Increased prevalence of pediatric pneumococcal serotypes in elderly adults," Clinical Infectious Diseases, vol. 41, no. 4, pp. 481-487, 2005.

[7] M. Imöhl, R. R. Reinert, and M. van der Linden, "Regional differences in serotype distribution, pneumococcal vaccine coverage, and antimicrobial resistance of invasive pneumococcal disease among German federal states," International Journal of Medical Microbiology, vol. 300, no. 4, pp. 237-247, 2010.

[8] R. R. Reinert, A. Queck, A. Kaufhold, M. Kresken, and R. Lütticken, "Antimicrobial resistance and type distribution of Streptococcus pneumoniae isolates causing systemic infections in Germany, 1992-1994," Clinical Infectious Diseases, vol. 21, no. 6, pp. 1398-1401, 1995.

[9] R. R. Reinert, A. Al-Lahham, M. Lemperle et al., "Emergence of macrolide and penicillin resistance among invasive pneumococcal isolates in Germany," Journal of Antimicrobial Chemotherapy, vol. 49, no. 1, pp. 61-68, 2002.

[10] R. Von Kries, A. Siedler, H. J. Schmitt, and R. R. Reinert, "Proportion of invasive pneumococcal infections in German children preventable by pneumococcal conjugate vaccines," Clinical Infectious Diseases, vol. 31, no. 2, pp. 482-487, 2000.

[11] R. R. Reinert, S. Haupts, M. van der Linden et al., "Invasive pneumococcal disease in adults in North-Rhine Westphalia, Germany, 2001-2003," Clinical Microbiology and Infection, vol. 11, no. 12, pp. 985-991, 2005.

[12] A. Kaufhold, R. Lütticken, and J. Henrichsen, "Capsular types and antibiotic susceptibility of Streptococcus pneumoniae isolated from patients with systemic infections in West Germany," European Journal of Clinical Microbiology, vol. 6, no. 6, pp. 696-697, 1987.

[13] S. Rückinger, R. von Kries, R. R. Reinert, M. van der Linden, and A. Siedler, "Childhood invasive pneumococcal disease in Germany between 1997 and 2003: variability in incidence and serotype distribution in absence of general pneumococcal conjugate vaccination," Vaccine, vol. 26, no. 32, pp. 3984-3986, 2008.

[14] D. Foster, K. Knox, A. S. Walker et al., "Invasive pneumococcal disease: epidemiology in children and adults prior to implementation of the conjugate vaccine in the Oxfordshire region, England," Journal of Medical Microbiology, vol. 57, no. 4, pp. 480-487, 2008.

[15] J. Flamaing, J. Verhaegen, J. Vandeven, N. Verbiest, and W. E. Peetermans, "Pneumococcal bacteraemia in Belgium (19942004): the pre-conjugate vaccine era," Journal of Antimicrobial Chemotherapy, vol. 61, no. 1, pp. 143-149, 2008.

[16] H. B. Konradsen and M. S. Kaltoft, "Invasive pneumococcal infections in Denmark from 1995 to 1999: epidemiology, serotypes, and resistance," Clinical and Diagnostic Laboratory Immunology, vol. 9, no. 2, pp. 358-365, 2002.

[17] M. Imöhl, R. R. Reinert, and M. van der Linden, "Adult invasive pneumococcal disease between 2003 and 2006 in North-Rhine Westphalia, Germany: serotype distribution before recommendation for general pneumococcal conjugate vaccination for children <2 years of age," Clinical Microbiology and Infection, vol. 15, no. 11, pp. 1008-1012, 2009.

[18] M. C. de Cunto Brandileone, D. V. V. Simonsen, S. Tadeu Casagrande, et al., "Characteristics of isolates Streptococcus pneumoniae from middle-aged and elderly adults in Brazil: capsular serotypes and antimicrobial sensitivity to invasive infections," Brazilian Journal of Infectious Diseases, vol. 2, pp. 90-96, 1998.

[19] J. F. Plouffe, S. K. Moore, R. Davis, and R. R. Facklam, "Serotypes of Streptococcus pneumoniae blood culture isolates from adults in Franklin County, Ohio," Journal of Clinical Microbiology, vol. 32, no. 6, pp. 1606-1607, 1994.

[20] C. G. Whitney, M. M. Farley, J. Hadler et al., "Increasing prevalence of multidrug-resistant Streptococcus pneumoniae in the United States," New England Journal of Medicine, vol. 343, no. 26, pp. 1917-1924, 2000.

[21] R. Lütticken and A. Kaufhold, "Serotypen und Antibiotikaempfindlichkeit von Streptococcus pneumoniae (Pneumokokken) im Raum Köln," Immunitat und Infektion, vol. 13, no. 3, pp. 99-107, 1985.

[22] L. A. Hicks, L. H. Harrison, B. Flannery et al., "Incidence of pneumococcal disease due to non- pneumococcal conjugate vaccine (PCV7) serotypes in the United States during the era of widespread PCV7 vaccination, 1998-2004," Journal of Infectious Diseases, vol. 196, no. 9, pp. 1346-1354, 2007.

[23] R. R. Reinert, "The antimicrobial resistance profile of Streptococcus pneumoniae," Clinical Microbiology and Infection, vol. 15, supplement 3, pp. 7-11, 2009.

[24] W. P. Hausdorff, "Invasive pneumococcal disease in children: geographic and temporal variations in incidence and serotype 
distribution," European Journal of Pediatrics, vol. 161, supplement 2, pp. S135-S139, 2002.

[25] S. Rückinger, M. van der Linden, R. R. Reinert, R. von Kries, F. Burckhardt, and A. Siedler, "Reduction in the incidence of invasive pneumococcal disease after general vaccination with 7-valent pneumococcal conjugate vaccine in Germany," Vaccine, vol. 27, no. 31, pp. 4136-4141, 2009.

[26] S. I. Aguiar, I. Serrano, F. R. Pinto, J. Melo-Cristino, and M. Ramirez, "Changes in Streptococcus pneumoniae serotypes causing invasive disease with non-universal vaccination coverage of the seven-valent conjugate vaccine," Clinical Microbiology and Infection, vol. 14, no. 9, pp. 835-843, 2008.

[27] R. Dias and M. Caniça, "Invasive pneumococcal disease in Portugal prior to and after the introduction of pneumococcal heptavalent conjugate vaccine," FEMS Immunology and Medical Microbiology, vol. 51, no. 1, pp. 35-42, 2007.

[28] A. Lepoutre, E. Varon, S. Georges, L. Gutmann, and D. Lévy-Bruhl, "Impact of infant pneumococcal vaccination on invasive pneumococcal diseases in France, 2001-2006," Euro Surveillance, vol. 13, no. 35, 2008.

[29] R. Mera, L. A. Miller, T. R. Fritsche, and R. N. Jones, "Serotype replacement and multiple resistance in Streptococcus pneumoniae after the introduction of the conjugate pneumococcal vaccine," Microbial Drug Resistance, vol. 14, no. 2, pp. 101-107, 2008.

[30] D. Akduman, J. M. Ehret, and F. N. Judson, "Comparison of secular trends in pneumococcal serotypes causing invasive disease in Denver, Colorado (1971-2004) and serotype coverage by marketed pneumococcal vaccines," Clinical Microbiology and Infection, vol. 12, no. 11, pp. 1141-1143, 2006.

[31] W. P. Hausdorff, J. Bryant, P. R. Paradiso, and G. R. Siber, "Which pneumococcal serogroups cause the most invasive disease: implications for conjugate vaccine formulation and use-part I," Clinical Infectious Diseases, vol. 30, no. 1, pp. 100-121, 2000.

[32] Ständige Impfkommission (STIKO), "Empfehlungen der Ständigen Impfkommission (STIKO) am Robert KochInstitut (Stand Juli 2006):Begründungen zur allgemeinen Empfehlung der Impfungen gegen Pneumokokken- und Meningokokken im Säuglings- und Kindesalter," Epidemiolgisches Bulletin, vol. 30, pp. 255-260, 2006. 

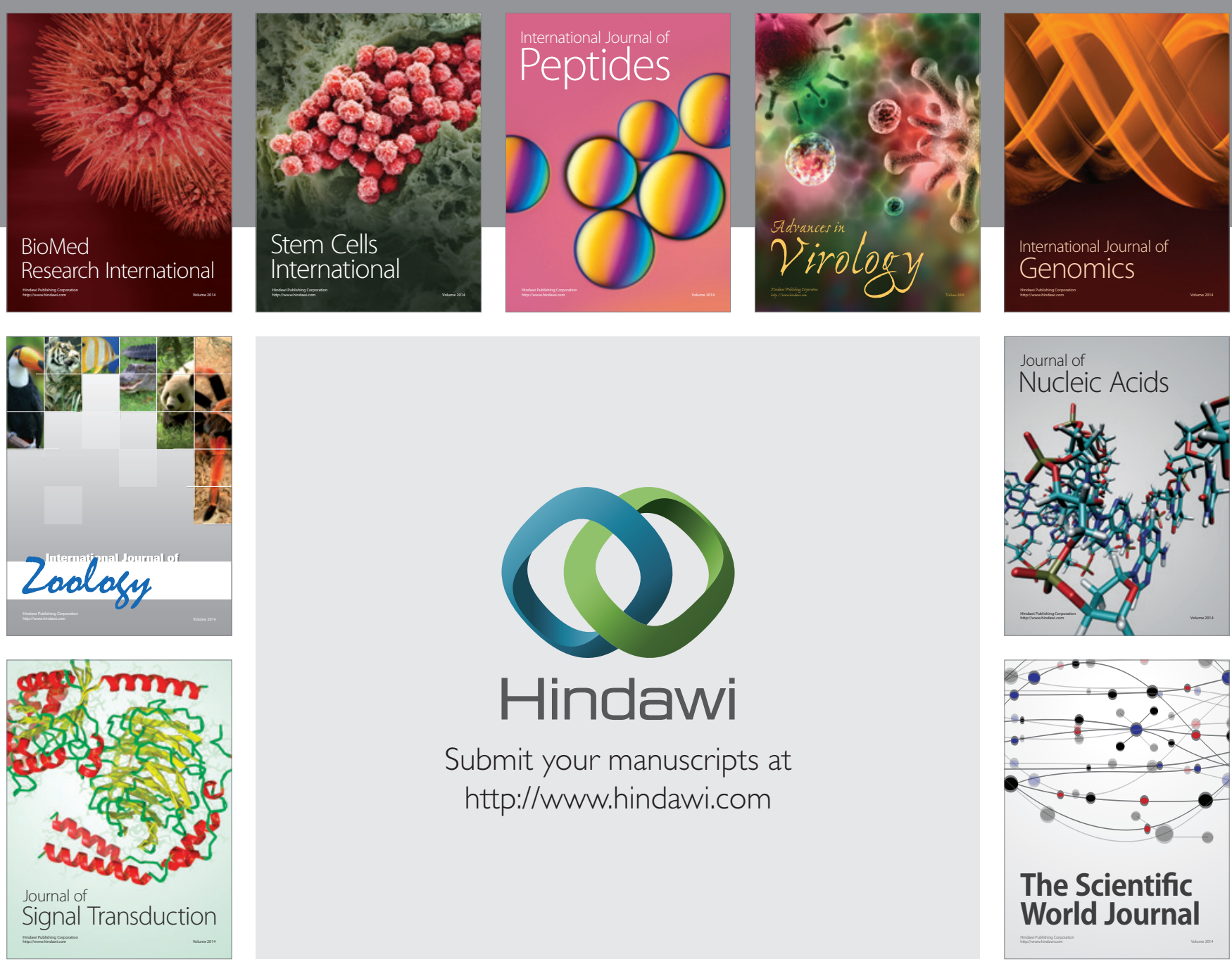

Submit your manuscripts at

http://www.hindawi.com
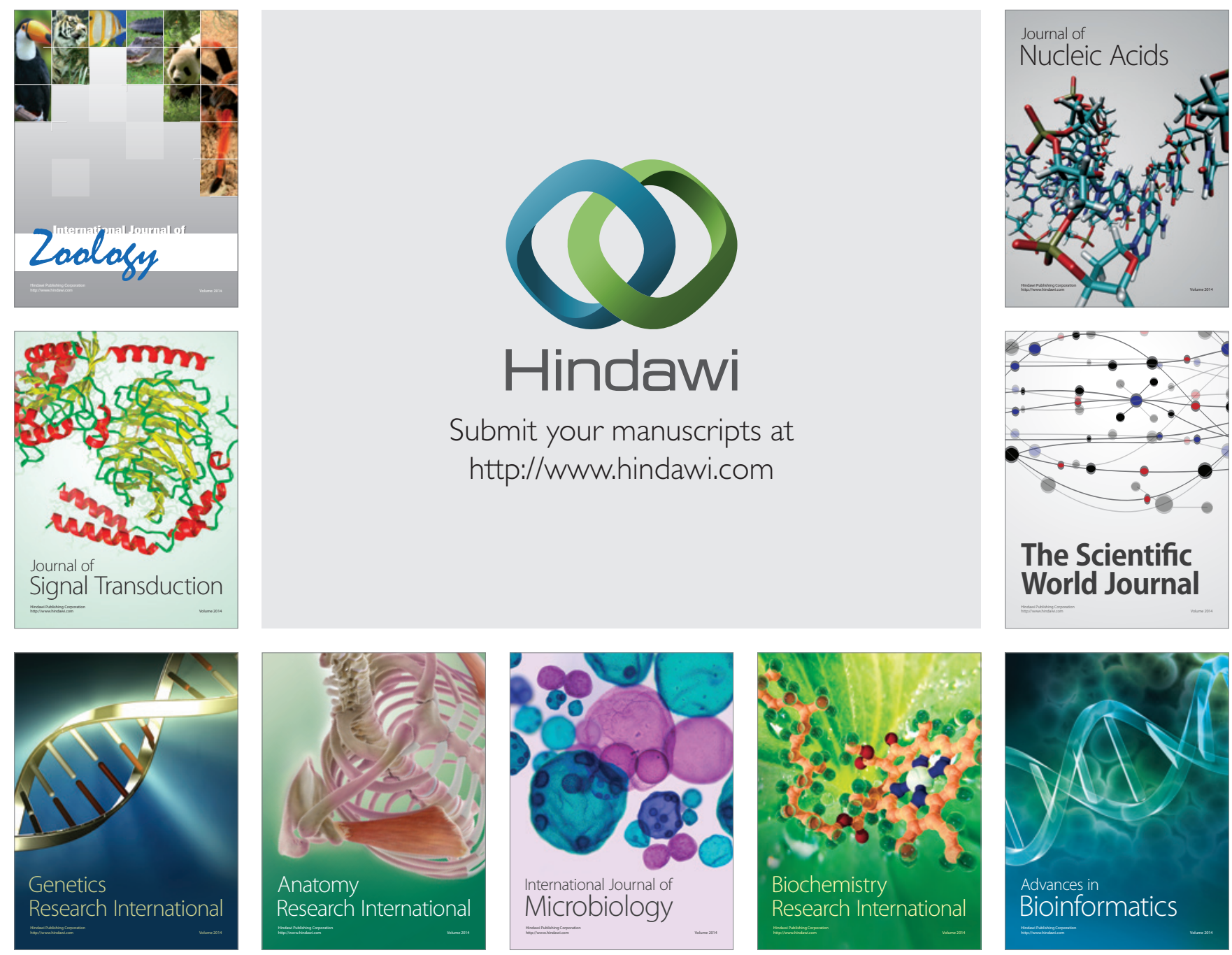

The Scientific World Journal
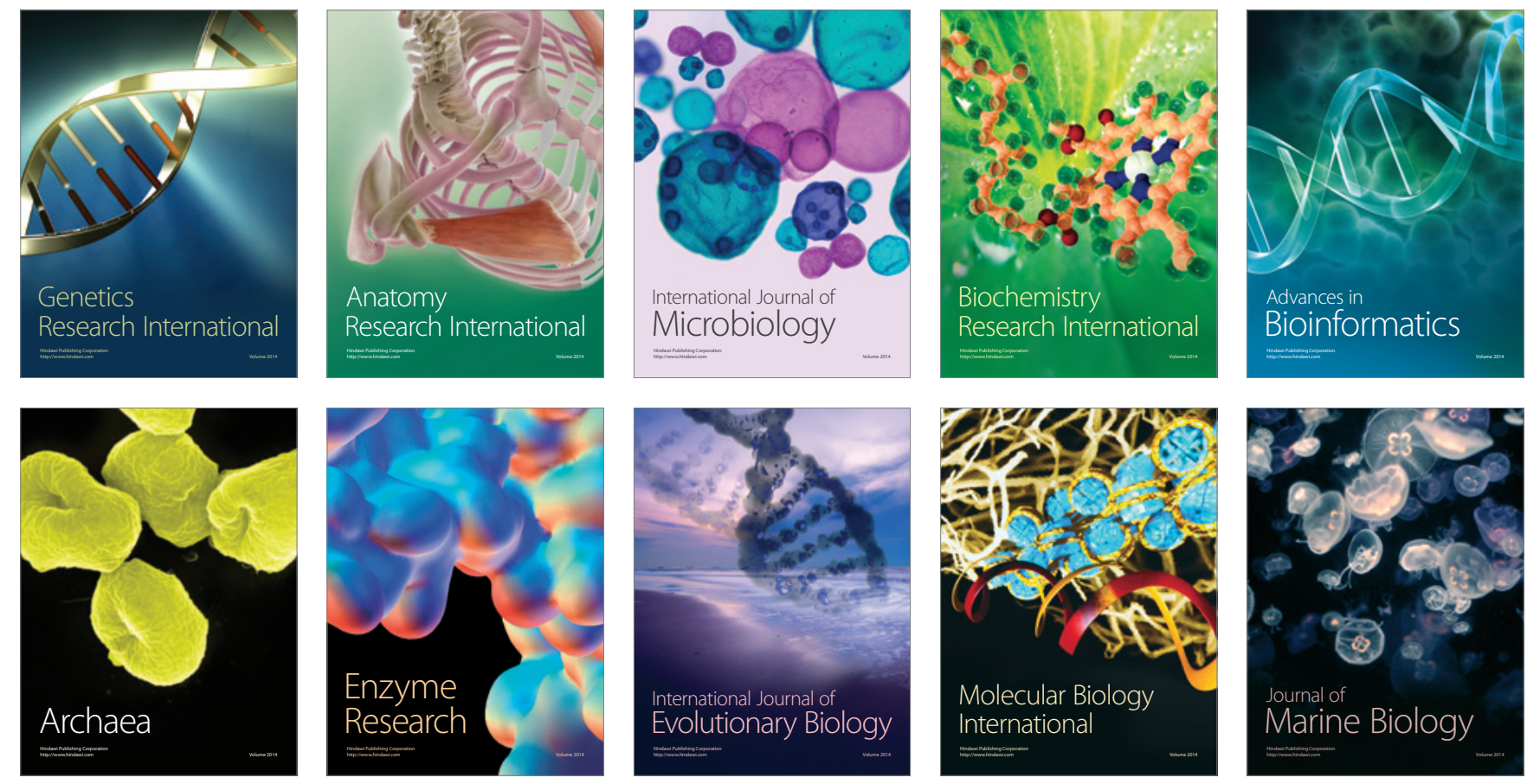